\title{
A Modelling of the Coupled Thermodiffuso-Elastic Linear Behaviour. Application to Explosive Decompression of Polymers
}

\author{
G. Rambert ${ }^{1}$, J.C. Grandidier ${ }^{1}$, L. Cangémi² and Y. Meimon ${ }^{2}$ \\ 1 Laboratoire de mécanique et physique des matériaux, EN SM A, 1, avenue Ader, BP 40109, 86961 Futuroscope Cedex - France \\ 2 Institut français du pétrole, 1 et 4, avenue de Bois-Préau, 92852 Rueil-M almaison C edex - France \\ e-mail: rambert@Impm.ensma.fr - grandidier@Impm.ensma.fr - laurent.cangemi@ifp.fr - yves.meimon@ifp.fr
}

\begin{abstract}
Résumé - Modélisation du comportement thermodiffuso-élastique linéaire couplé. Application à la décompression explosive de polymères - Une modélisation thermodiffuso-mécanique est proposée ici dans le but de décrire le comportement couplé de polymères initialement placés dans un environnement gazeux et sujets à une décompression «explosive». Plusieurs descriptions du volume élémentaire représentatif (VER) pouvant répondre à cet objectif sont détaillées. La modélisation est ensuite conduite sur la représentation la plus simple du VER comme un mélange homogène de polymère et de gaz. Dans le cadre de la thermodynamique des milieux standard généralisés, les lois constitutives couplées sont établies en se limitant à un comportement mécanique élastique linéaire. L'implantation de ce modèle dans le logiciel ABAQUS ${ }^{\mathrm{TM}}$ a alors permis de mener une première étude qualitative des effets de couplages directs dont les principaux résultats sont discutés.
\end{abstract}

\footnotetext{
Abstract-A Modelling of the Coupled Thermodiffuso-Elastic Linear Behaviour. Application to Explosive Decompression of Polymers - A thermodiffuso-mechanical modelling is proposed in this article with the aim of describing the coupled behaviour of polymers, which are initially placed in a gaseous environment and subjected to an "explosive" decompression. Some descriptions of the elementary representative volume (ERV) are considered and detailed. The modelling is then developed from the simplest representation, which consists in describing the ERV as a homogenous mixture of polymer and gas. In the framework of generalized standard media, coupled constitutive equations are only proposed for a linear elastic behaviour. The numerical implementation of this model in ABAQUS ${ }^{\mathrm{TM}}$ led to a first qualitative study of the direct couplings. Its main results are discussed here.
} 


\section{NOTATIO NS}

\section{General and Mathematical Notations}

$\star \quad$ quantity associated to the mixture

$\star_{0} \quad$ initial quantity associated to the mixture

$\star_{g}\left(\star_{p}\right)$ quantity associated to gas (polymer)

$\star_{g}^{0}\left(\star_{g}^{0}\right)$ initial quantity associated to gas (polymer)

$\vec{\star}^{g} \quad$ vector

$\star \quad$ second order tensor

$\underline{\underline{I}} \quad$ identity tensor

$\left(\frac{\mathrm{d}}{\mathrm{d} t}\right) \quad$ time derivative

$\left(\frac{\partial}{\partial}\right)_{\star} \quad$ partial derivative at $\star$ constant

grad gradient operator

div divergence operator

tr trace operator

$\vec{\star} \cdot \overrightarrow{\text { scalar product }}$

\section{Thermodynamic Notations}

$\psi \quad$ specific free energy $\left(\mathrm{J} \cdot \mathrm{kg}^{-1}\right)$

s $\quad$ specific entropy $\left(\mathrm{J} \cdot \mathrm{K}^{-1} \cdot \mathrm{kg}^{-1}\right)$

$\mathbf{S}_{0} \quad$ initial specific entropy $\left(\mathrm{J} \cdot \mathrm{K}^{-1} \cdot \mathrm{kg}^{-1}\right)$

$\vec{J} S \quad$ entropy flux $\left(\mathrm{W} \cdot \mathrm{K}^{-1} \cdot \mathrm{m}^{-2}\right)$

$\Phi \quad$ volume dissipation $\left(\mathrm{W} \cdot \mathrm{m}^{-3}\right)$

$\Phi_{1} \quad$ volume dissipation linked to temperature gradient $\left(\mathrm{W} \cdot \mathrm{m}^{-3}\right)$

$\Phi_{2} \quad$ volume dissipation linked to chemical potentials gradient $\left(\mathrm{W} \cdot \mathrm{m}^{-3}\right)$

$d \quad$ dissipation potential $\left(\mathrm{W} \cdot \mathrm{m}^{-3}\right)$

$d^{*}, d_{i}^{*} \quad$ dual dissipation potentials $\left(\mathrm{W} \cdot \mathrm{m}^{-3}\right)$

\section{Mechanical N otations}

$\begin{array}{ll}u_{r} & \begin{array}{l}\text { radial displacement }(\mathrm{m}) \\ \text { acceleration of the considered particle in the } \\ \text { mixture }\left(\mathrm{m} \cdot \mathrm{s}^{-2}\right)\end{array} \\ \vec{f} & \begin{array}{l}\text { body force per unit of mass }\left(\mathrm{N} \cdot \mathrm{kg}^{-1}\right) \\ \text { total Cauchy stress tensor }(\mathrm{Pa})\end{array} \\ \underline{\underline{\sigma}} & \text { initial total Cauchy stress tensor }(\mathrm{Pa}) \\ \underline{\underline{\sigma}}_{0} & \text { infinitesimal elastic strain tensor } \\ \frac{\underline{\varepsilon}^{e}}{\lambda}, \mu & \text { Lamé coefficients }(\mathrm{Pa}) \\ \mathrm{E} & \text { Young coefficient }(\mathrm{Pa}) \\ v & \text { Poisson coefficient }\end{array}$

\section{Thermal N otations}

$T \quad$ absolute temperature (K)

$T_{0} \quad$ initial absolute temperature (K)
$\vec{J}_{q} \quad$ heat flux per unit area $\left(\mathrm{W} \cdot \mathrm{m}^{-2}\right)$

$r \quad$ volume density of heat generated by an external source $\left(\mathrm{W} \cdot \mathrm{m}^{-3}\right)$

$\Lambda \quad$ thermal conductivity $\left(\mathrm{W} \cdot \mathrm{m}^{-1} \cdot \mathrm{K}^{-1}\right)$

$C_{\underline{\underline{\varepsilon}}, c_{i}} \quad$ specific heat at constant strain and normalized concentrations $\left(\mathrm{J} \cdot \mathrm{K}^{-1} \cdot \mathrm{kg}^{-1}\right)$

\section{Diffusion N otations}

$\rho \quad$ average mixture density $\left(\mathrm{kg} \cdot \mathrm{m}^{-3}\right)$

$c_{i} \quad$ normalized concentration of the $i^{\text {th }}$ constituent (Pa)

$c_{i}^{0} \quad$ initial normalized concentration of the $i^{\text {th }}$ constituent $(\mathrm{Pa})$

$\mu_{i} \quad$ mass chemical potential of the $i^{\text {th }}$ constituent $\left(\mathrm{J} \cdot \mathrm{kg}^{-1}\right)$

$\mu_{i}^{0} \quad$ initial mass chemical potential of the $i^{\text {th }}$ constituent $\left(\mathrm{J} \cdot \mathrm{kg}^{-1}\right)$

$\vec{J}_{m_{i}} \quad$ relative mass flux of the $i^{\text {th }}$ constituent $\left(\mathrm{kg} \cdot \mathrm{m}^{-2} \cdot \mathrm{s}^{-1}\right)$

$s_{i} \quad$ solubility coefficient of the $i^{\text {th }}$ constituent in the mixture $\left(\mathrm{Pa}^{-1}\right)$

D diffusivity $\left(\mathrm{m}^{2} \cdot \mathrm{s}^{-1}\right)$

\section{Coupling Notations}

$\alpha_{T} \quad$ isotropic heat expansion coefficient $\left(\mathrm{K}^{-1}\right)$

$\alpha_{c} \quad$ coefficient of expansion linked to diffusion phenomenon

$k_{\mu} \quad$ coefficient linked to the chemical potential gradient effect on the gas mass flux $\left(\mathrm{kg} \cdot \mathrm{s} \cdot \mathrm{m}^{-3}\right)$

$k_{T} \quad$ coefficient accounting for the thermal gradient effect on the entropy flux $\left(\mathrm{W} \cdot \mathrm{m}^{-1} \cdot \mathrm{K}^{-2}\right)$

$c_{T \mu} \quad$ coefficient corresponding to the temperature (chemical potential) gradient effect on the mass (entropy) flux $\left(\mathrm{kg} \cdot \mathrm{m}^{-1} \cdot \mathrm{s}^{-1} \cdot \mathrm{K}^{-1}\right)$

d coefficient representing the effect of temperature variation (concentration variation) on the chemical potential (entropy) $\left(\mathrm{J} \cdot \mathrm{kg}^{-1} \cdot \mathrm{K}^{-1}\right)$

D coefficient defined as $\left(\rho \mathrm{D} / k_{\mu}\right)\left(\mathrm{m}^{2} / \mathrm{s}^{2}\right)$.

\section{INTRODUCTIO N}

During the past twenty years, the use of polymers expanded widely in technical structures. In petroleum industry for instance, they form sheaths and gaskets used for oil transportation. They are exposed to severe in-service environment, defined by high temperature $\left(80\right.$ to $\left.150^{\circ} \mathrm{C}\right)$ and by a gas pressure, which fluctuates abruptly between high $(100 \mathrm{MPa})$ and low values. These drops in pressure (called explosive decompression) sometimes induce irreversible damage in the polymer as a result of a full coupling between mechanical, thermal and diffusion phenomena. Depending 
on the nature of the gas-polymer system, decompression rate and thermal conditions, such damage appears either locally (mesocracking) or in a diffuse way (foaming). Predictive tools, taking these couplings into account, should be used to foresee the in-service behaviour of such structures. The work in progress is based on the development of constitutive laws. The latter would make it possible to report the different interactions between mechanical, thermal and diffusion phenomena.

From an experimental viewpoint, the explosive decompression phenomenon was often studied, particularly in the case of elastomers, which are often employed in the oil industry and are not very resistant to explosive decompression. A few examples are given hereafter for information: the works of Briscoe et al. (1992, 1993, 1994), Campion (1990) and Liatsis (1989). General investigations were also made on thermoplastics by Dewimille et al. (1993) and Jarrin et al. (1994), more particularly on polyethylene in a methane environment (Gaillard-Devaux, 1995) and polyvinylidene fluoride with carbon dioxide (Lorge et al., 1999). All these studies confirm the existence of a strong coupling between mechanical, thermal and diffusion phenomena.

From a modelling viewpoint, the theoretical description of these coupling phenomena is generally based on an empirical or thermodynamic interpretation of experiments. These interactions are taken into account at two levels: in the expression of polymer characteristics as a function of pressure, temperature and concentration on the one hand, in the thermodynamic formulation of the model on the other hand, that correspond to the indirect and direct couplings respectively.

The indirect couplings were specially considered in the literature. A number of molecular models, based on the polymer internal structure, represent the diffusion characteristics variation with pressure, concentration and/or temperature. Some bibliographical syntheses are given on this subject by Liatsis (1989), Gaillard-Devaux (1995) and more recently by Klopffer and Flaconnèche (2001). In the same way, there is some information on mechanical and thermal characteristics. For instance, Rodier-Renaud (1994) define the isobaric expansion coefficient as a function of temperature for polyethylene. Nevertheless, the complexity of mechanisms, the multiplicity of dependences and the difficulty in carrying out characterization tests under high gas pressure do not make it possible to define all the indirect couplings and often lead to complicated expressions of these couplings with limited validity domain.

The direct couplings were tackled in the classical framework of thermodynamics of irreversible processes. They are taken into account via a direct reliance of thermodynamic potentials (specific free energy, dissipation potential) on all the variables (mechanical, thermal, diffusion). This second approach of couplings enables one to represent successfully a number of physical phenomena. To give just one example, it is easy for Soret effect (i.e. the case in which a thermal gradient induces a mass flux) to be described (Papon and Leblond, 1990). Cailletaud et al. (2002) presented a bibliographical work on this direct way of considering couplings. In this paper, metallic material behaviour has been studied, especially the interaction between metal oxidation and mechanical load. In polymer mechanics, interesting theoretical developments, which led to the DNLR (distributions of non-linear relaxations) model, were also proposed by Cunat (1991) and fellow workers. A distribution of relaxation processes is used to represent the internal dissipation phenomena. At any time, the system tends towards a state of equilibrium in accordance with some physical considerations. This formulation makes it possible to take account of a lot of direct couplings (mechanical, magnetic, thermal, etc.). While this modelling thermodynamic framework is well established, nevertheless the relaxation spectra definition is delicate when couplings are present and was not considered in the explosive decompression case. So, actually there is no clearly defined model in which these direct couplings are thoroughly considered.

Faced with this situation, a modelling which takes the direct couplings as well as the indirect couplings into account should be developed in the long term. It is a prerequisite when one aims at foreseeing the damage incipience. The first step, which is the subject of this article, is to establish a model in which only the direct couplings are considered. The material is considered at the macroscopic scale in the framework of thermodynamics of continuous media with internal variables.

In the first part of this document, we propose some ideas on the elementary representative volume (ERV) description. Five alternatives are considered. They differ in the way of describing the polymer internal structure on the one hand, and in the way of describing the gas transport phenomenon on the other hand. Then, the simplest one is taken as a starting point of thermodiffuso-mechanical modelling, in other words the ERV is considered to be a homogenous polymer and gas mixture. Gas diffusion is a molecular diffusion, which obeys a Fick's type law and thus is driven by concentration gradients. We look for the easiest approach to the direct couplings that is why a classical thermodynamic (for mechanic) framework is chosen. The thermodynamic potentials employed are quadratic forms so that the equations of state as well as the complementary evolution laws are linear. This model does not account for the indirect couplings, which means that material characteristics have constant values. The second part of this article deals with a particular version namely the linear thermodiffuso-elastic model.

Its numerical implementation in ABAQUS ${ }^{\mathrm{TM}}$ led to a first qualitative study of the direct couplings. The structure under consideration, corresponding to an infinite pipe, was subjected to mechanical, thermal and diffusion loads. The effects of coupling on its elastic response are presented in the third part of the document. 
Firstly, this numerical tool will enable one to estimate the contribution made by such a modelling to the explosive decompression study. Admittedly this modelling is limited, but such a tool taking account of the interaction between mechanics, thermic and diffusion will facilitate the comprehension of the basic phenomena. It is an obligatory stage if one wishes, in the long run, to approach damage phenomena. Secondly, the simulations will lead to a new protocol of experimental characterizations, which will in the long term make it possible to distinguish the effect of the direct couplings from the effect of indirect couplings due to the intrinsic dependences of polymer characteristics.

\section{CHARACTERIZATION OF THE ERV}

In this development, the thermodiffuso-mechanical behaviour of polymers is described at a macroscopic scale in the framework of the thermodynamics of continuous media with internal variables. The physical "poverty" of this modelling is filled in by a more or less complicated kinematics, which leads to a relevant representation of the microstructure. Any ERV is represented by a mono- or biphasic entity, in which gas transport is due to the concentration and/or pressure gradients. So, five alternatives were considered, corresponding to an increasingly elaborate approach to the strain internal mechanisms. Interest here is in the various modelling which could be developed within the framework of polymer mechanics.

\subsection{ERV Description Possibilities}

\subsubsection{Monophasic Modelling}

First of all, the polymer is described as a monophasic medium, which is particularly appropriate to amorphous materials such as thermosetting elastomers and uncrystallized thermoplastics. The gas transport corresponds to a molecular diffusion and/or a flow. These different perceptions of transport phenomena lead to the three models, which are presented below.

\section{Homogeneous Mixture}

In the first model, gas diffuses within the material according to a Fick's type law, that is to say under the effect of the concentration gradients. Then, the ERV is described as a classical continuous medium, made up of a homogeneous polymer-gas mixture. The equations of state as well as the complementary evolution laws relate to this mixture (Fig. 1).

Damage phenomena can be approached only with internal variables, which have to be numerous enough to account for the damage modes observed during an explosive decompression.

\section{Saturated Porous Medium with an Impermeable Skeleton}

In the second model, gas flows within the material according to a Darcy's type law, i.e. under the effect of pressure gradients. So, the ERV is considered as a saturated porous medium in which the polymer corresponds to the impermeable skeleton and the gas saturates the connected porous space (Fig. 2).

In this case, damage processes can be taken into account classically by some internal variables but also by a mechanical effect of gas-polymer interaction corresponding to the internal structure effect (pre-existing defects). Thus, this second ERV representation contains more potentialities for the modelling of damage than the first one. It is a different physical description, which employs some geometrical variables associated with a microscopic scale (i.e. porosity and tortuosity). The presence of these interconnected cavities is debatable at a mesoscopic scale because they are not observable. However, it is conceivable at a lower scale. One can imagine that gas molecules contained in these cavities are numerous enough to be governed by classical equations of state (PVT) as in a porous medium. Some questions remain about interconnectivity, tortuosity and free volume notion. Besides, the treatment of the initial state (i.e. before absorption) poses also a problem. As the "void" is not conceivable in the connected porous space, the nature of the gas, which is present at the initial state, has to be defined from physical considerations.

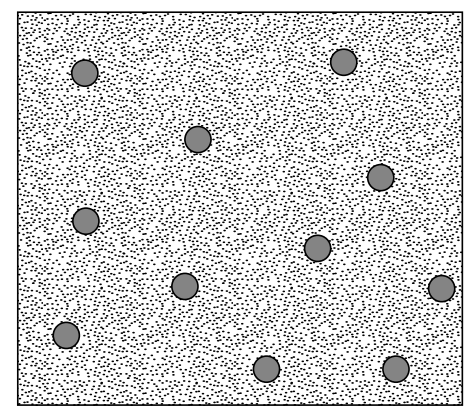

Figure 1

Homogeneous mixture

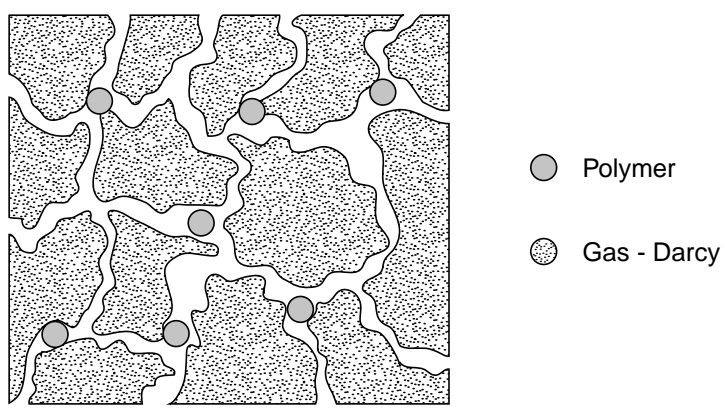

Figure 2

Saturated porous medium with an impermeable skeleton. 
Such a modelling was already considered in the literature. For instance, a thermoporo-elastic model was proposed by Weiler (1992) to study the rocket ablative materials such as carbon-phenolics. The formulation suggested by Coussy (1991) was also applied in the context of the thermoporoelastic behaviour of rocks (Onaisi, 1991) and the thermohydro-mechanical one (Skoczylas et al., 1992). All these studies highlighted some effects of coupling.

\section{Saturated Porous Medium with a Permeable Skeleton}

In the third model, the gas transport corresponds to a molecular diffusion within the skeleton (i.e. according to a Fick's type law) and also a flow within the connected porous space (i.e. according to a Darcy's type law). The ERV is described as in the previous case, except that now the polymer, which forms the skeleton, is permeable to the gas (Fig. 3).
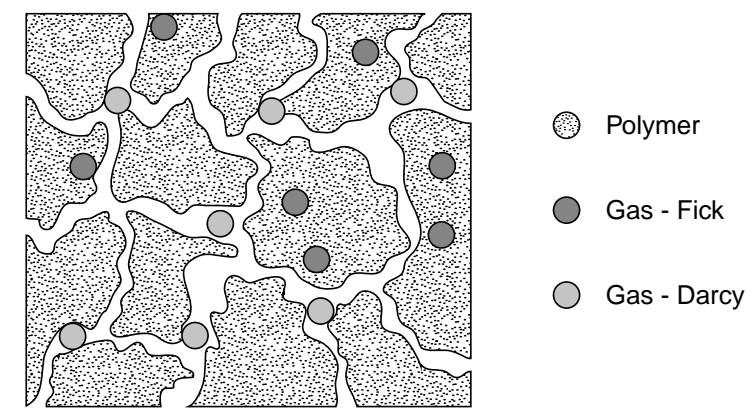

Figure 3

Saturated porous medium with a permeable skeleton.

The gas is absorbed in the skeleton on time scales which are different from those associated to the outflow within the connected porous space. That is why this modelling can describe the hysteresis effects and non-linearities observed during the diffusion tests. Moreover, this approach makes it possible to account for the complicated evolutions of the polymer characteristics with impregnation. Finally, different damage mechanisms can be considered according to the interaction between gas and polymer.
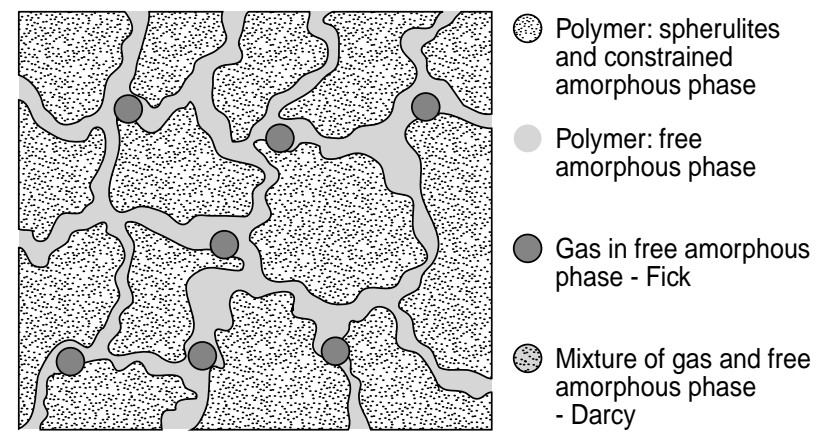

Figure 4

Unsaturated porous medium with an impermeable skeleton.

\subsubsection{Biphasic Modelling}

In the framework of semi-crystalline polymers, the ERV representation can be improved by taking account of the polymer morphology. Cangémi and Meimon (2001) proposed an advanced mechanical model in which the polymer is described as a biphasic or porous medium. Each constituent has its own constitutive law. The spherulites and the constrained amorphous phase form the skeleton whereas the free amorphous phase defines the "fluid". This last flows within the connected porous space of each ERV. This modelling led to a satisfactory representation of the polymer mechanical behaviour.

The introduction of diffusion and coupling requires further developments. Studies made by the IFP under gaseous pressure corresponding to an explosive decompression, showed that the gas diffused mainly in the free amorphous phase. Nevertheless, it is very probable that under higher pressure, gas diffuses also within the constrained amorphous phase associated to the spherulites. Then, two alternatives are considered.

Unsaturated Porous Medium with an Impermeable Skeleton In the fourth model, gas diffuses within the free amorphous phase according to a Fick's type law and the homogenous mixture of gas and free amorphous phase flows within the connected porous space, governed by a Darcy's type law.

The ERV is still described as a porous medium filled now with the mixture of gas and free amorphous phase (Fig. 4). So, the problem of the initial state (i.e. before absorption) does not arise any more. This representation contains interesting morphological characteristics. Even if the description of the polymer microstructure is poor, it offers great potentialities in the differentiation of damage modes.

Unsaturated Porous Medium with a Permeable Skeleton In this last model, the gas diffuses also between the crystalline lamellae (i.e. in the constrained amorphous phase linked to the spherulites) according to a Fick's type law. Indeed, the skeleton is assumed to be permeable to the gas. Each component of the mixture has to be considered separately (Fig. 5).
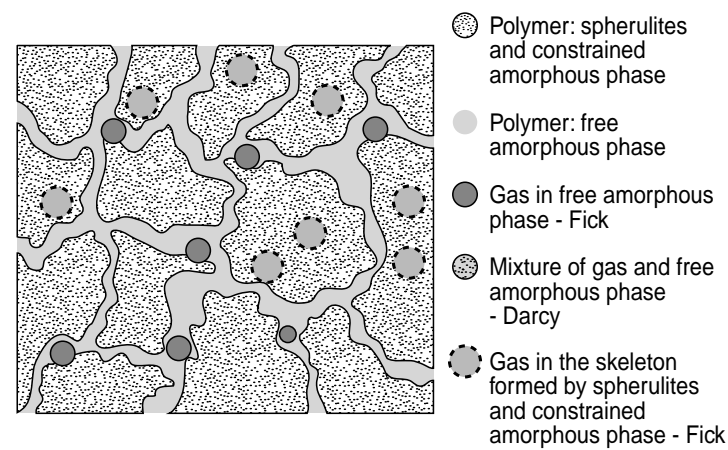

Figure 5

Unsaturated porous medium with an permeable skeleton. 
Such a modelling will probably describe some strong nonlinearities and will constitute an improvement in the damage treatment as well as in the approach of the impregnation effects.

\subsection{Modelling Choice}

Increasingly complicated descriptions of the ERV have been proposed. If couplings were specially considered in the framework of the porous media, it is less usual in the case of mixtures framework. Besides, the semi-crystalline polymer is described as a porous medium in which the "fluid" corresponds to a mixture of free amorphous phase and gas.

Thus, the mixtures framework is chosen to develop the thermodiffuso-mechanical model. Such a modelling will enable one to approach the amorphous polymer behaviour subjected to an explosive decompression. Moreover, it is a prerequisite work when one aims at modelling the semicrystalline behaviour.

\section{THERMO DIFFUSO -ELASTIC MO DELIN G}

\subsection{Thermodynamic Framework}

The model is developed in the framework of the generalized standard media and therefore follows a classical approach. A field of state variables represents the material at any time. The introduction of a first potential (specific free energy) makes it possible to set laws linking thermodynamic forces (stress, entropy, chemical potential) to dual variables (strain, temperature, normalized concentration). The dissipations, which are only associated to thermal and diffusion phenomena in an elastic behaviour case, are discussed using a second potential (dissipation potential). This potential expression depends on the considered coupling assumption (total or not). Once defined, it leads to complementary evolution laws (Fourier's, Fick's). These different steps are presented in the following paragraphs.

In this thermodynamic framework, every ERV is described at a macroscopic scale as a bicomponent, continuous, homogenous medium: polymer and gas. One focuses on the average behaviour of the mixture defined by average physical value (based on each species value). Moreover, this ERV is open and thus trades heat as well as matter with the surrounding medium. The two-species diffusion is a molecular diffusion, which obeys a Fick's type law and then is driven by concentration gradients. In addition, the material is assumed to be isotropic and is studied in the framework of small perturbations. Finally, a strong assumption consists in keeping constant each ERV characteristic in time and space. This approximation is by no means verified but makes it possible to simplify strongly the equations on the one hand and to account for only the direct couplings on the other hand.

\subsection{Local Equations}

The behaviour of polymers subjected to pressure, gas concentration as well as temperature variations is defined by diffusion, dynamic and thermal equations. The two former equation families are classically developed but the thermal one is not so easy since it contains couplings corresponding to dissipative phenomena.

\subsubsection{Mass Balance}

In a bicomponent system without chemical reaction, the mass of each species remains separately the same, which leads to two mass balance equations.

The two components of the mixture are characterized by the following variables:

$-\rho$ average mixture density $\left(\mathrm{kg} / \mathrm{m}^{3}\right)$;

$-c_{i}$ normalized concentration of the $i^{\text {th }}$ constituent $(\mathrm{Pa})$, defined as the concentration (mass fraction, i.e. $\mathrm{kg} / \mathrm{kg}$ ) and solubility si ratio $\left(\mathrm{Pa}^{-1}\right)$;

- $\vec{J}_{m_{i}}$ relative mass flux of the $i^{\text {th }}$ diffusing phase $\left(\mathrm{kg} / \mathrm{m}^{2} \cdot \mathrm{s}\right)$.

The mass balance for the $i^{\text {th }}$ component leads to the following diffusion equation:

$$
\rho s_{i} \frac{\mathrm{d} c_{i}}{\mathrm{~d} t}=-\operatorname{div} \vec{J}_{m_{i}}
$$

This classical balance equation is applied to polymer and gas. Nevertheless, mass flux and normalized concentrations satisfy by definition the following equations:

$$
\vec{J}_{m_{g}}=-\vec{J}_{m_{p}} \text { and } s_{g} \frac{\mathrm{d} c_{g}}{\mathrm{~d} t}=-s_{p} \frac{\mathrm{d} c_{p}}{\mathrm{~d} t}
$$

So, both the diffusion equations are linked and thus only the gas one will be used further in this paper:

$$
\rho s_{g} \frac{\mathrm{d} c_{g}}{\mathrm{~d} t}=-\operatorname{div} \vec{J}_{m_{g}}
$$

\subsubsection{Mechanical Balance}

The mechanical balance equation is derived from the fundamental principle of dynamics. When one neglects the inertia effects $(\rho \vec{\gamma} \approx \overrightarrow{0}$ ), it leads to the following static equation:

$$
\overrightarrow{\operatorname{div}} \underline{\underline{\sigma}}+\rho \vec{f}
$$

where $\underline{\underline{\sigma}}$ is the total Cauchy stress tensor (Pa), $\vec{f}$ is the body force per unit of mass $(\mathrm{N} / \mathrm{kg})$ at any point within the ERV and $\vec{\gamma}$ the considered particle acceleration $\left(\mathrm{m} / \mathrm{s}^{2}\right)$.

\subsubsection{Thermodynamic Balance - First and Second Laws}

Thermodynamic Potential, Equations of State

In the context of thermodynamics of continuous media, one classically employs the specific free energy $\psi$ of the mixture $(\mathrm{J} / \mathrm{kg})$. For an elastic behaviour, this potential depends on four state variables: the temperature $T(\mathrm{~K})$, the infinitesimal 
strain tensor $\underline{\underline{\varepsilon}}^{e}$ and the gas and polymer normalized concentrations $\overline{\bar{c}}_{g}$ and $c_{p}$, respectively:

$$
\psi=\psi\left(\underline{\underline{\varepsilon}}^{e}, T, c_{g}, c_{p}\right)
$$

When a normal configuration is assumed and the first two thermodynamics laws are considered, constitutive laws also known as equations of state can be derived from $\psi$ as follows:

$$
\underline{\underline{\sigma}}=\rho\left(\frac{\partial \Psi}{\partial \underline{\underline{\varepsilon}}^{e}}\right)_{T, c_{g}, c_{p}}, \mathbf{s}=-\left(\frac{\partial \Psi}{\partial T}\right)_{\underline{\underline{\varepsilon}}^{e}, c_{g}, c_{p}} \text { and } \mu_{i}=\frac{1}{s_{i}}\left(\frac{\partial \Psi}{\partial c_{i}}\right)_{T, \underline{\underline{\varepsilon}}, c_{k \neq i}}
$$

where $\mathbf{s}(\mathrm{J} / \mathrm{kg} \cdot \mathrm{K})$ denotes the specific entropy and $\mu_{i}(\mathrm{~J} / \mathrm{kg})$ the mass chemical potential for the $i^{\text {th }}$ species (dual variable of the mass fraction).

Remark: $\left(\frac{\partial}{\partial}\right)_{\star}$ means partial derivation with $\star$ as constant value.

Under the assumption of small perturbations, that is to say infinitely small strains and small differences of temperature and normalized concentration, the development of the potential $\psi$ to the second order leads to the following linear constitutive equations:

$$
\begin{aligned}
\underline{\underline{\sigma}=\underline{\sigma}_{0}} & +\lambda\left(\operatorname{tr} \underline{\underline{\varepsilon}}^{e}\right) \underline{\underline{I}}+2 \mu \underline{\underline{\varepsilon}}^{e} \\
& -(3 \lambda+2 \mu)\left[\alpha_{T}\left(T-T_{0}\right)+\alpha_{c} s_{g}\left(c_{g}-c_{g}^{0}\right)\right] \underline{\underline{I}} \\
\mathbf{s}=\mathbf{s}_{0} & +\frac{1}{T_{0}} C_{\underline{\underline{\varepsilon}}^{e}, c_{i}}\left(T-T_{0}\right) \\
& +\frac{1}{\rho}(3 \lambda+2 \mu) \alpha_{T} \operatorname{tr} \underline{\underline{\varepsilon}}^{e}+\mathbf{d} s_{g}\left(c_{g}-c_{g}^{0}\right) \\
\mu_{g}-\mu_{p}=\left(\mu_{g}^{0}-\mu_{p}^{0}\right)+\mathbf{D} s_{g}\left(c_{g}-c_{g}^{0}\right) & -\frac{1}{\rho}(3 \lambda+2 \mu) \alpha_{c} \operatorname{tr} \underline{\underline{\varepsilon}}^{e}-\mathbf{d}\left(T-T_{0}\right)
\end{aligned}
$$

where:

$-T_{0}, c_{g}^{0}, \underline{\sigma}_{0}, \mathbf{s}_{0}$ and $\mu_{i}^{0}$ respectively represent initial temperature, gas normalized concentration, stress, specific entropy and chemical potential of the $i^{\text {th }}$ constituent at zero strain, thermal and concentration variations;

$-C_{\underline{\underline{\varepsilon^{e}}, c_{i}}}=T\left(\frac{\partial \mathbf{s}}{\partial T}\right)_{\underline{\underline{\varepsilon^{e}}, c_{i}}}$ is the specific heat at constant strain and normalized concentration $(\mathrm{J} / \mathrm{kg} \cdot \mathrm{K})$;

$-\lambda$ and $\mu$ are the Lamé elastic coefficients $(\mathrm{Pa})$;

$-\alpha_{T}(\mathrm{~K})$ and $\alpha_{c}$ the isotropic expansion coefficients linked to respectively heat conduction and mass transport;

- the effect of temperature variation (concentration variation) on the chemical potential (entropy) is linked to the coefficient $\mathbf{d}(\mathrm{J} / \mathrm{kg} \cdot \mathrm{K})$;

- D is defined as a function of coefficients $\rho, D$ and $k_{\mu}$, the two latter ones being defined further in the document $\left(\mathrm{m}^{2} / \mathrm{s}^{2}\right)$;

$-\underline{\underline{I}}$ is the identity matrix.
Remark: tr represents the trace operator.

\section{Heat Equation}

Let $\vec{J}_{q}\left(\mathrm{~J} / \mathrm{m}^{2} \cdot \mathrm{s}\right)$ be the heat flux per unit area and $r\left(\mathrm{~J} / \mathrm{m}^{3} \cdot \mathrm{s}\right)$ the volume density of heat generated by an outer source (e.g. by an radiating source), the heat balance equation taking account of the different couplings has the following expression:

$$
\begin{aligned}
\rho C_{\underline{\underline{\varepsilon^{e}}}, c_{i}} \frac{\mathrm{d} T}{\mathrm{~d} t}=-\operatorname{div} \vec{J}_{q}+r & -(3 \lambda+2 \mu) \alpha_{T} T \operatorname{tr} \frac{\mathrm{d} \frac{\underline{\varepsilon}^{e}}{\mathrm{~d} t}}{} \\
& -\left[\left(\mu_{g}-\mu_{p}\right)+T \mathbf{d}\right] \rho s_{g} \frac{\mathrm{d} c_{g}}{\mathrm{~d} t}
\end{aligned}
$$

The last term corresponds to the temperature variation linked to gas diffusion within the polymer material.

\section{Dissipations}

The second law of thermodynamics makes it possible to define the volume dissipation $\Phi$ (positive or nil) linked to entropy generation. An elastic behaviour is characterized by the absence of internal variables, which leads to a nil intrinsic dissipation. $\Phi$ splits into two terms, $\Phi_{1}$ and $\Phi_{2}\left(\mathrm{~J} / \mathrm{m}^{3} \cdot \mathrm{s}\right)$, which are linked respectively to the temperature and chemical potential gradients, so that:

$$
\begin{gathered}
\Phi=\Phi_{1}+\Phi_{2} \geq 0, \quad \Phi_{1}=-\vec{J}_{s} \cdot \overrightarrow{\operatorname{grad}} T \\
\text { and } \Phi_{2}=-\vec{J}_{m_{g}} \cdot \overrightarrow{\operatorname{grad}}\left(\mu_{g}-\mu_{p}\right)
\end{gathered}
$$

where:

$$
\vec{J}_{s}=\frac{1}{T}\left[\vec{J}_{q}-\left(\mu_{g}-\mu_{p}\right) \vec{J}_{m_{g}}\right]
$$

is the entropy flux $\left(\mathrm{J} / \mathrm{s} \cdot \mathrm{K} \cdot \mathrm{m}^{2}\right)$.

Introduction of the dissipation potential $d$ and its dual $d^{*}$ - through Legendre-Fenchel transformation- $\left(\mathrm{J} / \mathrm{m}^{3} \cdot \mathrm{s}\right)$ is compatible a priori with the second law of thermodynamics. The expressions of these continuous, convex and positive functions depend on the considered coupling assumptions. Evolution equations are given for different $d^{*}$ in the following paragraph.

\section{Coupling Processing}

Under the assumption of a total coupling between dissipative phenomena, the dual potential becomes a unique function, which is given by Equation (7). In the framework of generalized standard media (i.e. normal dissipativity assumption), one sets the evolution complementary equations given by Equation (8).

$$
\begin{gathered}
d^{*}=d^{*}\left(-\overrightarrow{\operatorname{grad}} T,-\overrightarrow{\operatorname{grad}}\left[\mu_{g}-\mu_{p}\right]\right) \\
\vec{J}_{s}=\left(\frac{\partial d^{*}}{\partial[-\overrightarrow{\operatorname{grad}} T]}\right)_{-\operatorname{grad}\left(\mu_{g}-\mu_{p}\right)}, \vec{J}_{m_{g}}=\left(\frac{\partial d^{*}}{\partial\left[-\overrightarrow{\operatorname{grad}}\left(\mu_{g}-\mu_{p}\right)\right]}\right)_{-\operatorname{grad} T}
\end{gathered}
$$


Moreover, when the dissipation phenomena are assumed to be driven by linear and fully coupled laws, the potential $d^{*}$ is quadratic and leads in an isotropic case to the following laws:

$$
\begin{aligned}
& \vec{J}_{m_{g}}=-k_{\mu} \overrightarrow{\operatorname{grad}}\left[\mu_{g}-\mu_{p}\right]-c_{T \mu} \overrightarrow{\operatorname{grad}} T \\
& \vec{J}_{s}=-k_{T} \overrightarrow{\operatorname{grad}} T-c_{T \mu} \overrightarrow{\operatorname{grad}}\left[\mu_{g}-\mu_{p}\right]
\end{aligned}
$$

where:

$-k_{\mu}$ is linked to the chemical potential gradient effect on the gas mass flux $\left(\mathrm{kg} \cdot \mathrm{s} / \mathrm{m}^{3}\right)$;

$-k_{T}$ represents the thermal gradient effect on the entropy flux $\left(\mathrm{W} / \mathrm{m} \cdot \mathrm{K}^{2}\right)$;

$-c_{T \mu}$ is a coupling coefficient corresponding to the temperature (chemical potential) gradient effect on the mass (entropy) flux $(\mathrm{kg} / \mathrm{m} \cdot \mathrm{s} \cdot \mathrm{K})$.

With the previous expression of the chemical potential (Eq. 5), one obtains:

$$
\begin{aligned}
& \vec{J}_{m_{\mathrm{g}}}=-k_{\mu} \mathbf{D} s_{g} \overrightarrow{\operatorname{grad}} c_{g} \\
& +k_{\mu} \frac{(3 \lambda+2 \mu) \alpha_{c}}{\rho} \overrightarrow{\operatorname{grad}}\left[\operatorname{tr} \underline{\underline{\varepsilon}}^{e}\right]+\left(k_{\mu} \mathbf{d}-c_{T \mu}\right) \overrightarrow{\operatorname{grad}} T \\
& \vec{J}_{q}=-\left[\left(k_{T}-\mathbf{d} c_{T \mu}\right) T+\left(c_{T \mu}-\mathbf{d} k_{\mu}\right)\left(\mu_{g}-\mu_{p}\right)\right] \overrightarrow{\operatorname{grad} T} \\
& -\frac{D \rho}{k_{\mu}}\left[c_{T \mu} T+k_{\mu}\left(\mu_{g}-\mu_{p}\right)\right] s_{g} \overrightarrow{\operatorname{grad}} c_{g} \\
& +\frac{(3 \lambda+2 \mu) \alpha_{c}}{\rho}\left[c_{T \mu} T+k_{\mu}\left(\mu_{g}-\mu_{p}\right)\right] \overrightarrow{\operatorname{grad}}\left[\operatorname{tr} \underline{\underline{\varepsilon}}^{e}\right]
\end{aligned}
$$

When dissipative processes are assumed totally uncoupled, two dual potentials $d_{1}^{*}, d_{2}^{*}$, enter the expression of $d^{*}$ :

$$
d^{*}=d_{1}^{*}(-\overrightarrow{\operatorname{grad}} T)+d_{2}^{*}\left(-\overrightarrow{\operatorname{grad}}\left[\mu_{g}-\mu_{p}\right]\right)
$$

As previously stated, an important simplification of these equations consists in considering the dual dissipation potential as a quadratic form. One then obtains the well-known evolution equations, namely Fourier's (only conduction case) and Fick's (diffusion case) equations:

$$
\begin{gathered}
\vec{J}_{q}=-k_{T} T \overrightarrow{\operatorname{grad} T}, k_{T}=\frac{\Lambda}{T} \\
\text { and } \vec{J}_{m_{\mathrm{g}}}=-k_{\mu} \mathbf{D} s_{g} \overrightarrow{\operatorname{grad}} c_{g}, \mathbf{D}=\frac{\rho k_{\mu}}{\mathrm{D}}
\end{gathered}
$$

where $\Lambda$ is the thermal conductivity $(\mathrm{J} / \mathrm{m} \cdot \mathrm{s} \cdot \mathrm{K})$ and $\mathrm{D}$ the diffusivity $\left(\mathrm{m}^{2} / \mathrm{s}\right)$.

\section{Summary: Unknowns and Constitutive Equations}

The variables defining the thermodiffuso-mechanical state of the material (gas-polymer mixture) are the components of the displacement vector, the temperature $T$ and the gas normalized concentration $c_{g}$. Among all the different coefficients that were introduced, $k_{\mu}, c_{T \mu}$ and $\mathbf{d}$ are the only ones still to be determined through a parametric study.

With the previous equations, the thermodiffuso-elastic model is finally defined under a full coupling assumption as follows:

- Mechanical problem:

$$
\begin{aligned}
& \overrightarrow{\operatorname{div}} \underline{\underline{\sigma}}+\rho \vec{f}=\overrightarrow{0} \\
& \underline{\underline{\sigma}}=\underline{\underline{\sigma}}_{0}+\lambda\left(\operatorname{tr} \underline{\underline{\varepsilon}}^{e}\right) \underline{\underline{I}}+2 \mu \underline{\underline{\varepsilon}}^{e} \\
& -(3 \lambda+2 \mu)\left[\alpha_{T}\left(T-T_{0}\right)+\alpha_{c} 10^{6} s_{g}\left(c_{g}-c_{g}^{0}\right)\right] \underline{\underline{I}}
\end{aligned}
$$

- Diffusion problem:

$$
\begin{aligned}
& \rho 10^{6} s_{g} \frac{\mathrm{d} c_{g}}{\mathrm{~d} t}=\mathrm{D} \rho 10^{6} s_{g} \operatorname{div}\left(\overrightarrow{\operatorname{grad}} c_{g}\right) \\
& k_{\mu} \frac{(3 \lambda+2 \mu) \alpha_{c}}{\rho} \operatorname{div}\left(\overrightarrow{\operatorname{grad}}\left[\operatorname{tr} \underline{\underline{\varepsilon}}^{e}\right]\right)+\left(c_{T \mu}-k_{\mu} \mathbf{d}\right) \operatorname{div}(\overrightarrow{\operatorname{grad}} T)
\end{aligned}
$$

- Thermal problem (Eq. 11):

$$
\begin{aligned}
\rho C_{\underline{\underline{\varepsilon^{e}}, Y_{g}}} \frac{\mathrm{d} T}{\mathrm{~d} t} & =\left[\frac{\Lambda}{T}+\left(k_{\mu} \mathbf{d}-2 c_{T \mu}\right) \mathbf{d}\right] T \operatorname{div}(\overrightarrow{\operatorname{grad}} T)+r+\left(k_{\mu} \mathbf{d}-2 c_{T \mu}\right) \mathbf{d}(\overrightarrow{\operatorname{grad}} T)^{2} \\
& +\frac{1}{k_{\mu}}\left(\mathrm{D} \rho 10^{6} s_{g}\right)^{2}\left(\overrightarrow{\operatorname{grad}} c_{g}\right)^{2}-(3 \lambda+2 \mu) \alpha_{T} T \operatorname{tr} \frac{\mathrm{d} \underline{\underline{\varepsilon}}^{e}}{\mathrm{~d} t} \\
& -2 \mathrm{D} \rho 10^{6} s_{g} \frac{(3 \lambda+2 \mu) \alpha_{c}}{\rho} \overrightarrow{\operatorname{grad}}\left[\operatorname{tr} \underline{\underline{\varepsilon}}^{e}\right] \cdot \overrightarrow{\operatorname{grad}} c_{g}+k_{\mu}\left(\frac{(3 \lambda+2 \mu) \alpha_{c}}{\rho}\right)^{2}\left(\overrightarrow{\operatorname{grad}}\left[\operatorname{tr} \underline{\underline{\varepsilon}}^{e}\right]\right)^{2} \\
& +\left[c_{T \mu}-k_{\mu} \mathbf{d}\right] \frac{1}{k_{\mu}} \mathrm{D} \rho 10^{6} s_{g} T \operatorname{div}\left(\overrightarrow{\operatorname{grad}} c_{g}\right) \\
& -\left[c_{T \mu}-k_{\mu} \mathbf{d}\right] \frac{(3 \lambda+2 \mu) \alpha_{c}}{\rho} T \operatorname{div}(\overrightarrow{\operatorname{grad}}[\operatorname{tr} \underline{\underline{\varepsilon}}]) \\
& +2\left[c_{T \mu}-k_{\mu} \mathbf{d}\right] \frac{1}{k_{\mu}} \mathrm{D} \rho 10^{6} s_{g} \overrightarrow{\operatorname{grad}} c_{g} \cdot \overrightarrow{\operatorname{grad} T} \\
& -2\left[c_{T \mu}-k_{\mu} \mathbf{d}\right] \frac{(3 \lambda+2 \mu) \alpha_{c}}{\rho} \overrightarrow{\operatorname{grad}}\left[\operatorname{tr} \underline{\underline{\varepsilon}} \underline{\varepsilon}^{e}\right] \cdot \overrightarrow{\operatorname{grad}} T
\end{aligned}
$$




\section{QUALTATIVE STUDY OF COUPUNG}

The numerical implementation of the linear thermodiffusoelastic model in the software ABAQUSTM leads to a first qualitative study of direct couplings. The programmed elements are first validated considering an uncoupled behaviour. Then, couplings are introduced gradually and their effects on the polymer sheath of an infinite tube are given. The model contains five coefficients that define a more or less complicated coupling between mechanical, thermal and diffusion phenomena: $\alpha_{T}, \alpha_{c}, k_{\mu}, c_{T \mu}$ and $\mathbf{d}$. The $k_{\mu}$ parameter is studied first, because it cannot be eliminated. Secondly, the more classical coefficients of expansion, $\alpha_{T}$ and $\alpha_{c}$, are introduced. $c_{T \mu}$ and $\mathbf{d}$ parameters are considered finally.

\subsection{Structure and Loading}

The structure considered is a flexible pipe used for carbon dioxide transportation and formed by a polymer sheath. Its dimensions as well as the loading path and the boundary conditions are chosen so that the assumption of plane strain can be considered. Due to symmetries, only one quarter of the pipe section is represented by 3000 8-node quadrilaterals (Fig. 6).

All except the three unknown coupling coefficients $\left(k_{\mu}, c_{T \mu}\right.$ and $\left.\mathbf{d}\right)$ correspond to the polyvinylidene fluoride (PVDF) characteristics. They are measured at (Tie Bi, 2000) or extrapolated to (Flaconnèche et al., 2001) a temperature around $21^{\circ} \mathrm{C}$ (Table 1). The coefficient of expansion due to gas diffusion is determined at $130^{\circ} \mathrm{C}$ (data issued from recent tests, IFP). It is a relatively rare data in the literature because it requires specific experimental systems. Nevertheless, such an approximation of the characteristics values can be admitted because of the exploratory nature (in some aspects) of this study. All these material characteristics are assumed to be constant during the simulation.

The loading path is defined as corresponding approximately to the engineering applications (Fig. 7). However, the thermal exchange between the transported fluid and the sheath is not taken into account, in particular during the explosive decompression, which induces a temperature drop inside the pipe and therefore a modification of the inside boundary conditions.

\subsection{Uncoupled Case}

In the uncoupled case, four of the five coupling parameters are equal to zero:

$$
\alpha_{T}=0, \alpha_{c}=0, c_{T \mu}=0 \text { and } \mathbf{d}=0
$$

The following simplified system of equations describes the problem:

- Mechanical problem:

$$
\overrightarrow{\operatorname{div}} \underline{\underline{\sigma}}+\rho \vec{f}=\overrightarrow{0} \text { with } \underline{\underline{\sigma}}=\underline{\underline{\sigma}}_{0}+\lambda\left(\operatorname{tr} \underline{\underline{\varepsilon}}^{e}\right) \underline{\underline{I}}+2 \mu \underline{\underline{\varepsilon}}^{e}
$$

- Diffusion problem:

$$
\frac{\mathrm{d} c_{g}}{\mathrm{~d} t}=\mathrm{D} \operatorname{div}\left(\overrightarrow{\operatorname{grad}} c_{g}\right)
$$

- Thermal problem:

$$
\rho C_{\underline{\varepsilon^{e}}, Y_{g}} \frac{\mathrm{d} T}{\mathrm{~d} t}=\Lambda \operatorname{div}(\overrightarrow{\operatorname{grad}} T)+r+\frac{1}{k_{\mu}}\left(\mathrm{D} \rho 10^{6} s_{g}\right)^{2}\left(\overrightarrow{\operatorname{grad}} c_{g}\right)^{2}
$$

As the coefficient $k_{\mu}$ appears in the denominator of the last term of Equation (14), it cannot be cancelled. So, it is chosen in such a manner that the effect of diffusion on thermal problem can be neglected. Specifically, for a $k_{\mu}$ around $10^{30} \mathrm{~kg} \cdot \mathrm{s} / \mathrm{m}^{3}$, one obtains the same results that those issued from the uncoupled mechanical, thermal and diffusion simulations of ABAQUS ${ }^{\mathrm{TM}}$. In other words, this value of $k_{\mu}$ can be considered as relevant to an uncoupled case.

\subsection{Diffusion Effect on Thermal Problem via $\mathbf{k}_{\mu}$}

$k_{\mu}$ appears above to be the only parameter necessarily different from zero. It is possible to make its effect negligible but not to suppress it. That is why it is the first one introduced in the model.

\section{Theoretical Aspects}

From a thermodynamic viewpoint, $k_{\mu}$ defined as an Onsager coefficient is necessarily positive. Some simulations are made for the following parameters set:

$$
0<k_{\mu} \leq 10^{30}, \quad \alpha_{T}=0, \quad \alpha_{c}=0, \quad c_{T \mu}=0 \text { and } \mathbf{d}=0
$$

In these conditions, the thermodiffuso-elastic model is characterized by the foregoing equations (Eqs. 12, 13, 14). Strictly speaking, taking account of $k_{\mu}$ only, mechanical as well as diffusion problems correspond to the truly uncoupled

TABLE 1

Characteristics of a PVDF with $\mathrm{CO}_{2}$ at $21^{\circ} \mathrm{C}$ (except $\alpha_{c}$ which is measured at $130^{\circ} \mathrm{C}$ )

\begin{tabular}{c|c|c|c|c|c|c|c|c}
\hline$\rho$ & $\mathrm{E}$ & $v$ & $\begin{array}{c}C_{\underline{\underline{\varepsilon^{e}}}, Y_{g}} \\
(\mathrm{~J} / \mathrm{kg} \cdot \mathrm{K})\end{array}$ & $\begin{array}{c}\Lambda \\
(\mathrm{W} / \mathrm{m} \cdot \mathrm{K})\end{array}$ & $\begin{array}{c}\alpha_{T} \\
\left(\mathrm{~K}^{-1}\right)\end{array}$ & $\begin{array}{c}s_{g} \\
\left(\mathrm{~Pa}^{-1}\right)\end{array}$ & $\begin{array}{c}\mathrm{D} \\
\left(\mathrm{m}^{2} / \mathrm{s}\right)\end{array}$ & $\begin{array}{c}\alpha_{c} \\
(\varnothing)\end{array}$ \\
\hline 1745 & 1743 & 0.38 & 1045 & 0.26 & $10^{-5}$ & $5.539 \cdot 10^{-3}$ & $9.8 \cdot 10^{-12}$ & $2.2 \cdot 10^{-13}$ \\
\hline
\end{tabular}



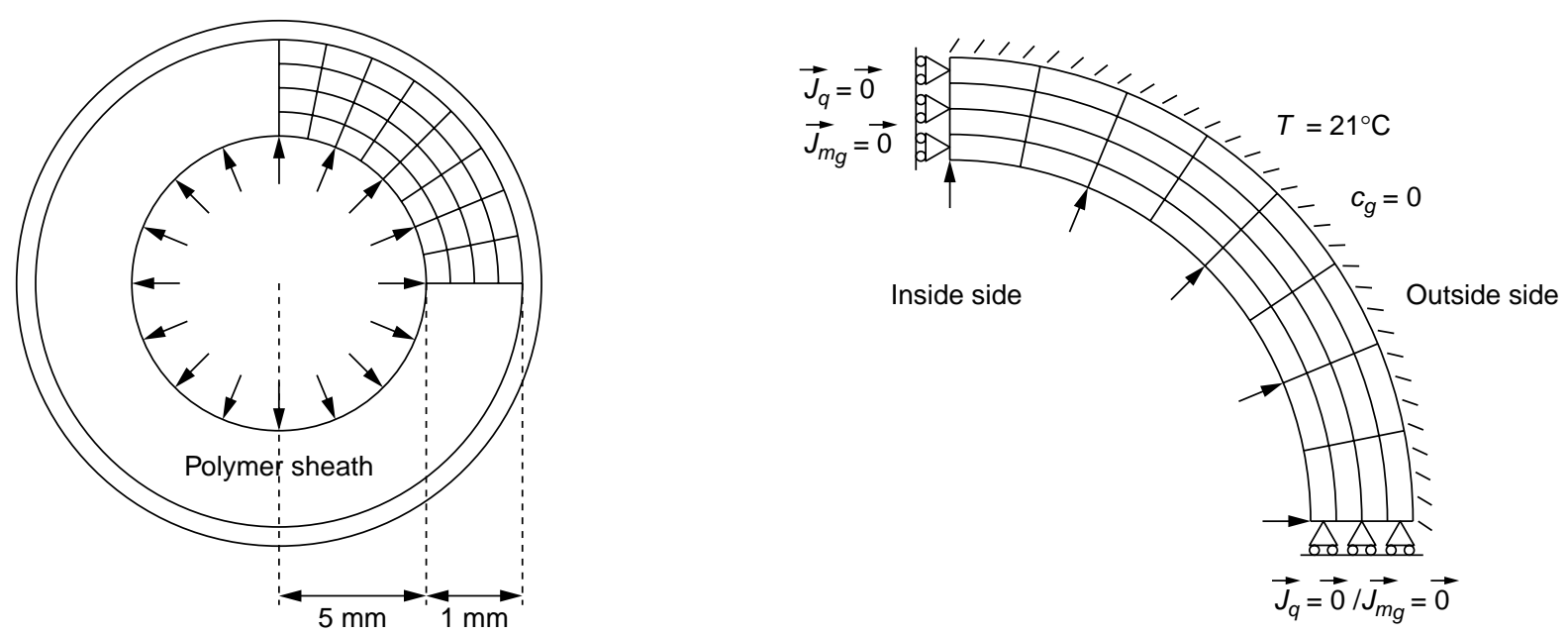

Figure 6

Section and meshing of the pipe.
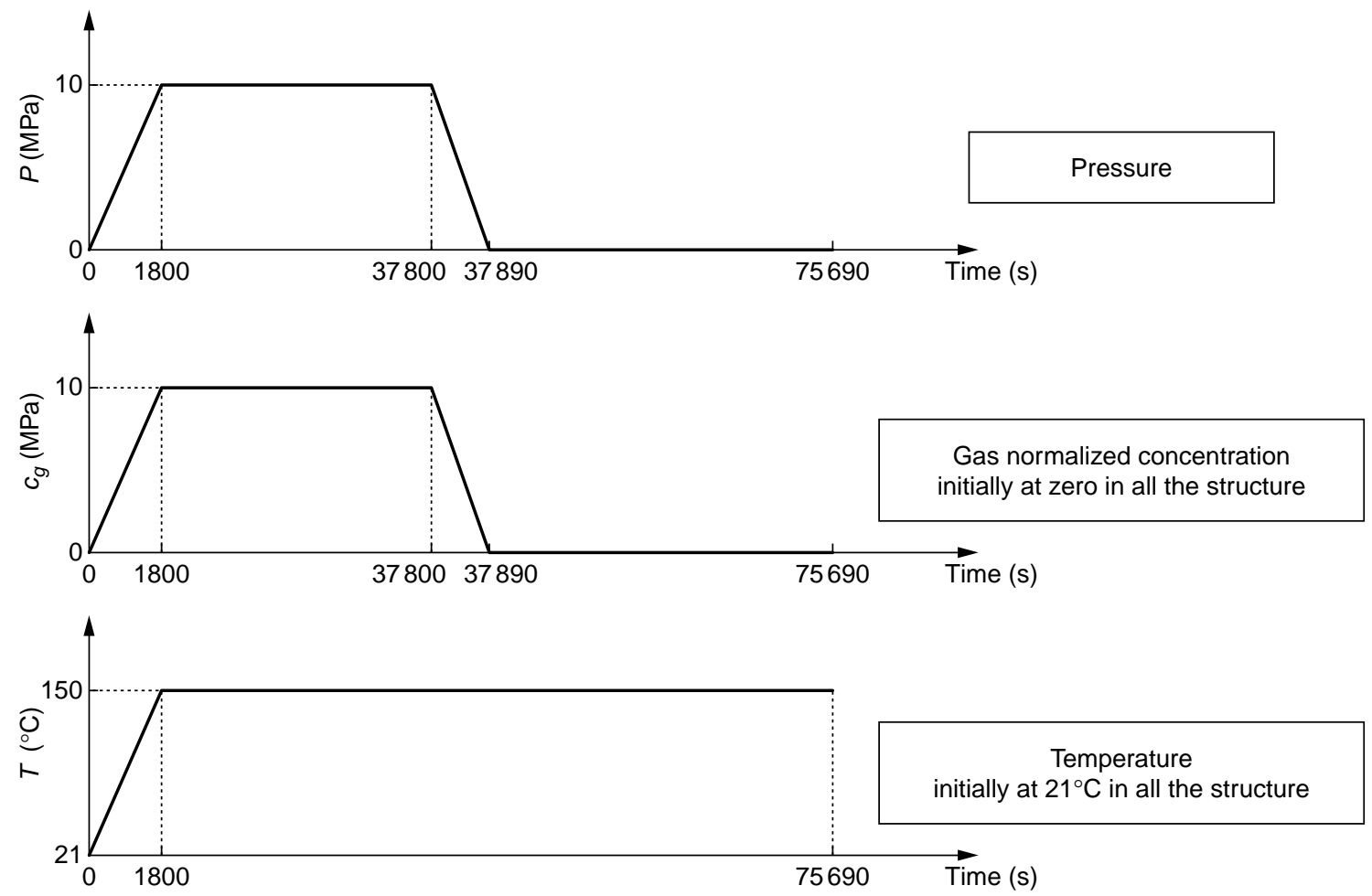

Figure 7

Boundary conditions and loading applied on the inside face of the pipe. 
case. The sole heat equation is modified: diffusion phenomenon induces now temperature variations. Thus, the only thermal response is studied.

\section{Numerical Aspects}

The effect of diffusion on thermal problem results in a local increase in temperature. This result was foreseeable because the coefficient $K_{\mu}$ relative to this effect is superior (or equal) to zero (Eq. 14).

The foregoing effect is negligible when the coefficient $k_{\mu}$ is between $10^{30}$ and $5 \cdot 10^{6} \mathrm{~kg} \cdot \mathrm{s} / \mathrm{m}^{3}$. Indeed, the relative variation of temperature compared to the uncoupled case is then lower to one percent. When the value of $k_{\mu}$ decreases further (below $5 \cdot 10^{6} \mathrm{~kg} \cdot \mathrm{s} / \mathrm{m}^{3}$ ), the coupling effect appears. The temperature rise is more important as $k_{\mu}$ is small, a result consistent with the heat equation $\left(k_{\mu}\right.$ appears to the denominator, Eq. 14). Moreover, a second threshold value occurs around $2 \cdot 10^{4} \mathrm{~kg} \cdot \mathrm{s} / \mathrm{m}^{3}$ : an inferior $k_{\mu}$ leads to disproportionate coupling effects with a relative variation of temperature around a hundred percent during the decompression. In the interval $\left[2 \cdot 10^{4} \mathrm{~kg} \cdot \mathrm{s} / \mathrm{m}^{3}, 5 \cdot 10^{6} \mathrm{~kg} \cdot \mathrm{s} / \mathrm{m}^{3}\right]$, this relative variation compared to the uncoupled situation remains more realistic, namely inferior to fifty percent.

To estimate this effect of diffusion on the thermal field, elements placed on a tube radius are followed during the simulation. Whatever the $k_{\mu}$ value, the maximal relative scatter of temperature is recorded at roughly 0.27 and $0.6 \mathrm{~mm}$ from the interior side during the compression and decompression phases, respectively (Fig. 8). This result is closely linked to the loading and boundary conditions corresponding to this simulation (Figs. 6 and 7).

During the first stage of the simulation (linearly increasing and constant concentration steps), the relative variation of temperature compared to the uncoupled case increases then decreases at a given point (Fig. 8). It reaches a maximal value faster at a point located nearer from the interior boundary than another one. This phenomenon can be explained by the evolution of the gas mass flux in space and time, in other words it is connected with the characteristic time of diffusion process. Moreover, a zone characterized by more complicated fluctuations of temperature appears on the radius owing to the nil concentration imposed on the outside edge of the structure. Indeed, this boundary condition results in strong gradients of concentration near the outside edge at an advanced stage in the diffusion phenomenon. For some particular values of $k_{\mu}$, the stationary state is not reached completely when decompression occurs.

During the second stage of the simulation (linearly decreasing and zero concentration steps), the heat increase is quite steady. The relative variation of temperature is maximal at the end of the linear unloading and then it tends towards zero (Fig. 8). Indeed, concentration gradients disappear as a nil concentration is imposed on each edge.
The trace of temperature curves at two points of a radius (Fig. 9) leads to the same observations.

\section{Physical Aspects}

This first "rudimentary" coupling describes the effect of diffusion on temperature evolution. Whatever the direction of diffusion is, the gas transport results in a local increase in the polymer temperature via $k_{\mu}$. From a physical viewpoint, such a temperature rise results from the evolution of polymer internal state, whose gas content varies with the pressure applied on the boundary.

\subsection{Introduction of Expansions}

The two other coefficients introduced represent the expansion linked to the variations of temperature $\left(\alpha_{T}\right)$ on the one hand and of concentration $\left(\alpha_{c}\right)$ on the other hand.

\subsubsection{Thermal Expansion}

First, only the thermal expansion coefficient is considered, which leads to the following configuration:

$$
10^{4} \leq k_{\mu} \leq 10^{30}, \alpha_{T} \neq 0, \alpha_{c}=0, c_{T \mu}=0 \text { and } \mathbf{d}=0
$$

\section{Theoretical Aspects}

Mechanical and thermal problems are thus modified. In the first one, thermal dilation occurs (Eq. 15) and in the second one, the rate of volumetric swelling strain induces a variation of temperature (Eq. 16). The diffusion equation remains the same as in the previous case (Eq. 13); nevertheless gas diffusion has an indirect effect on mechanics through the temperature field (Eq. 16). Indeed, diffusion acts on the temperature evolution via $k_{\mu}$, which has further repercussions on thermal expansion and thus on the mechanical problem. This last modifies finally the thermal problem through the variation of the rate of volumetric swelling strain.

$$
\begin{gathered}
\underline{\underline{\varepsilon}}^{e}=\underline{\varepsilon}_{0}+\frac{1+v}{\mathrm{E}} \underline{\underline{\sigma}}-\frac{v}{\mathrm{E}}(\operatorname{tr} \underline{\underline{\sigma}}) \underline{\underline{I}}+\alpha_{T}\left(T-T_{0}\right) \underline{\underline{I}} \\
\rho C_{{\underline{\underline{\varepsilon^{e}}}}_{,}, Y_{g}} \frac{\mathrm{d} T}{\mathrm{~d} t}=\Lambda \operatorname{div}(\overrightarrow{\operatorname{grad}} T)+r \\
+\frac{1}{k_{\mu}}\left(\mathrm{D} \rho 10^{6} s_{g}\right)^{2}\left(\overrightarrow{\operatorname{grad}} c_{g}\right)^{2}-(3 \lambda+2 \mu) \alpha_{T} T \operatorname{tr} \frac{\mathrm{d} \underline{\underline{\varepsilon}}^{e}}{\mathrm{~d} t}
\end{gathered}
$$

$\mathrm{E}(\mathrm{Pa})$ and $v$ being the coefficients of Young and Poisson, respectively.

\section{Numerical Aspects}

As the processes are relatively slow during the simulation, the temperature field is not affected by this classical coupling (which needs faster solicitations to be active).

The indirect effect of diffusion on mechanics results in a nonlinear distribution of volumetric swelling strain along 

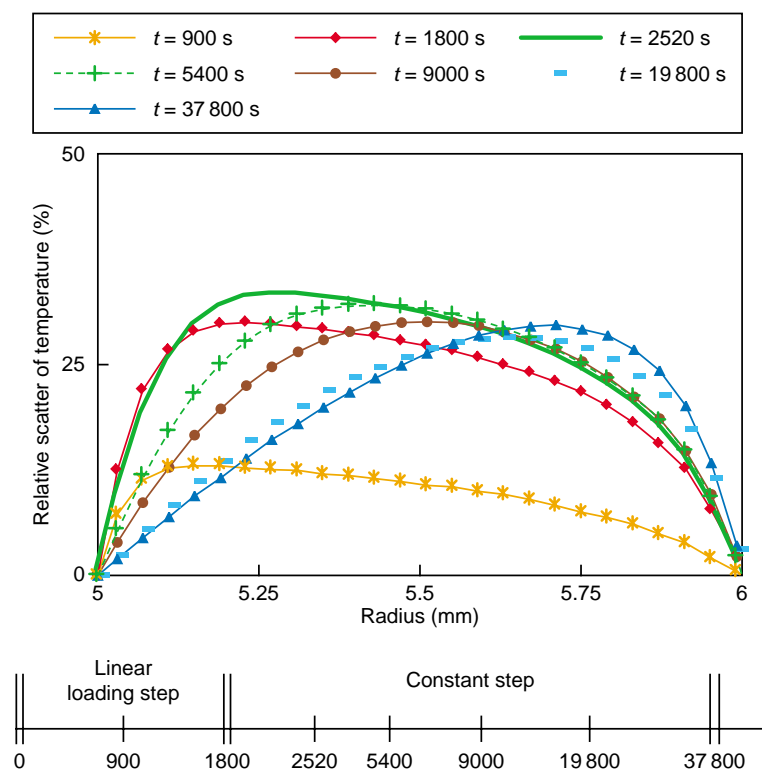

$37800 \quad 37810 \quad 3787037890$

Linear unloading step

\begin{tabular}{|c|c|c|c|c|}
\hline 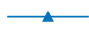 & $t=37810 \mathrm{~s}$ & $-\cdots+1=37870 \mathrm{~s}$ & - & $t=37890 \mathrm{~s}$ \\
\hline$\longrightarrow$ & $t=38070 \mathrm{~s}$ & $---+--t=38790 \mathrm{~s}$ & 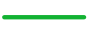 & $t=40410 \mathrm{~s}$ \\
\hline$\longrightarrow$ & $t=43290 \mathrm{~s}$ & $-\cdots--t=47970 \mathrm{~s}$ & — & $t=59490 \mathrm{~s}$ \\
\hline
\end{tabular}
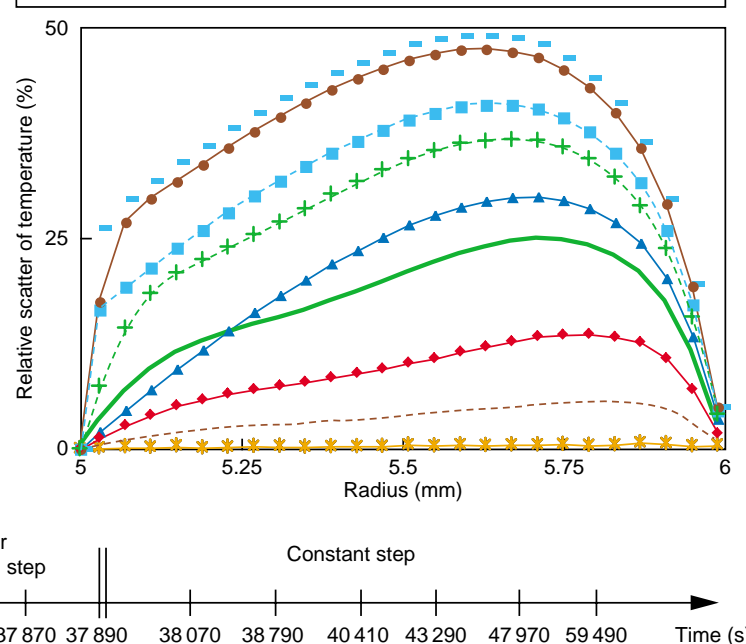

Constant step

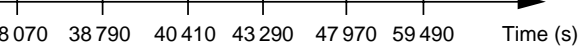

Figure 8

Spatial distribution of the relative scatter of temperature $\left(\left[T-T_{1}\right] /\left|T_{1}\right|\right)$, for the coupled case $(T)$ compared with the uncoupled case $\left(T_{1}\right)$ for $k_{\mu}=2 \cdot 10^{4} \mathrm{~kg} \cdot \mathrm{s} / \mathrm{m}^{3}$ at different time.
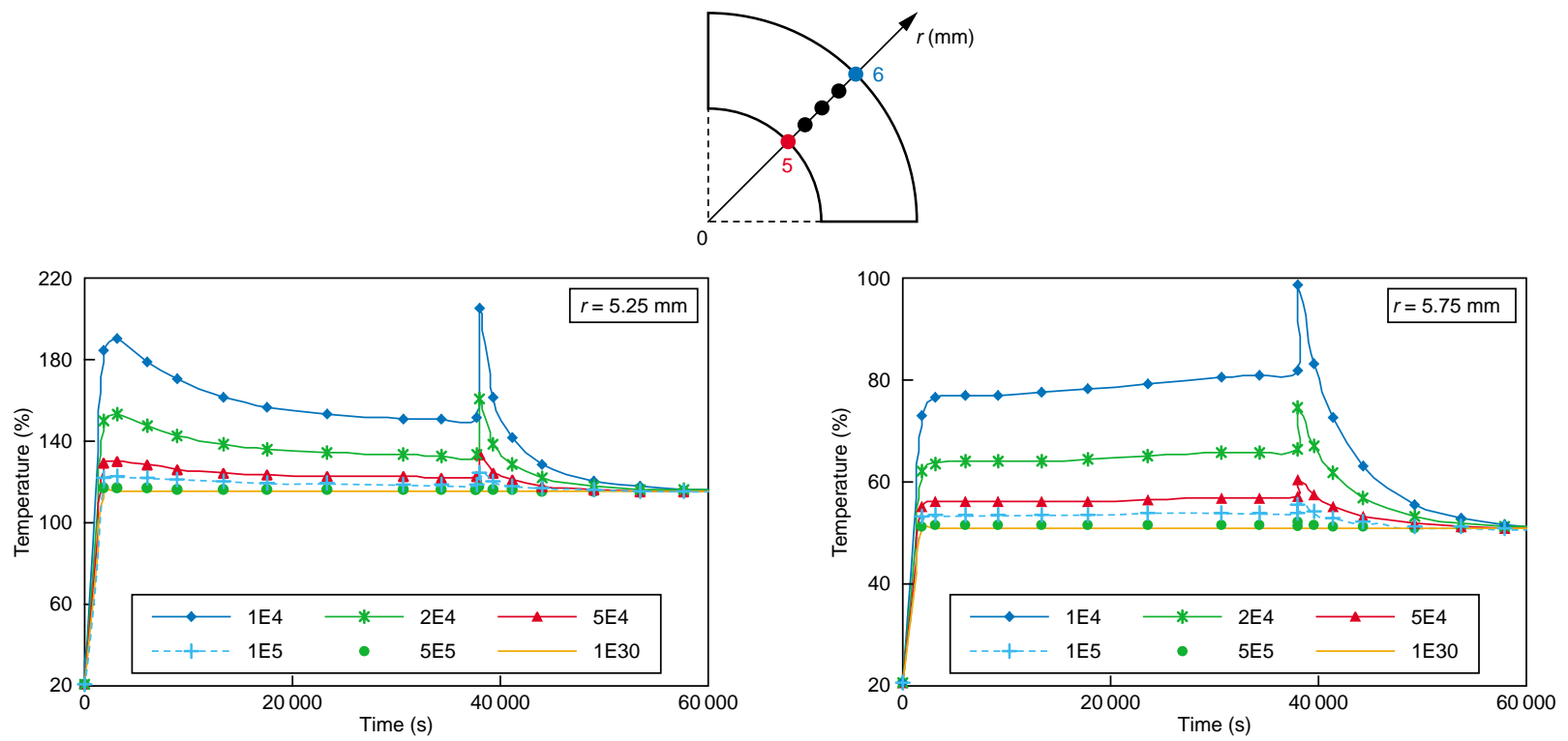

Figure 9

Temperature variation at two points of the radius for different $k_{\mu}$. 
a radius (Fig. 10). The profiles of thermal expansion given for a $k_{\mu}$ coefficient equal to $2 \cdot 10^{4} \mathrm{~kg} \cdot \mathrm{s} / \mathrm{m}^{3}$ (coupled case) and $10^{30} \mathrm{~kg} \cdot \mathrm{s} / \mathrm{m}^{3}$ (uncoupled case) show this phenomenon. Moreover, during the transient phases, a slight peak of strain appears near the interior edge of the structure. So, according to geometry and boundary conditions, one could eventually expect a localization of stress generated by coupling phenomena.

\subsubsection{Expansion Linked to Gas Diffusion}

At this step, the expansion coefficient $\alpha_{c}$ corresponding to the gas diffusion is introduced in the model:

$$
10^{4}<k_{\mu} \leq 10^{30}, \quad \alpha_{T} \neq 0, \quad \alpha_{c} \neq 0, \quad c_{T \mu}=0 \text { and } \mathbf{d}=0
$$

\section{Theoretical Aspects}

The problem is modified overall. In this case, the coupling between thermal and diffusion phenomena via $k_{\mu}$ is inevitable. Indeed, when a non-zero coefficient $\alpha_{c}$ (coefficient expansion due to diffusion) is considered, any plausible value of $k_{\mu}$ favours this effect of diffusion on temperature evolution. So, the basic model used to describe the thermodiffuso-mechanical behaviour of polymers contains necessarily all the couplings considered in this paragraph.

The values of $k_{\mu}$ are chosen so that the concentration evolution is governed mainly by the classical term linked to the divergence of the concentration gradient. This condition leads to:

$$
10^{4}<k_{\mu} \leq 10^{10}
$$
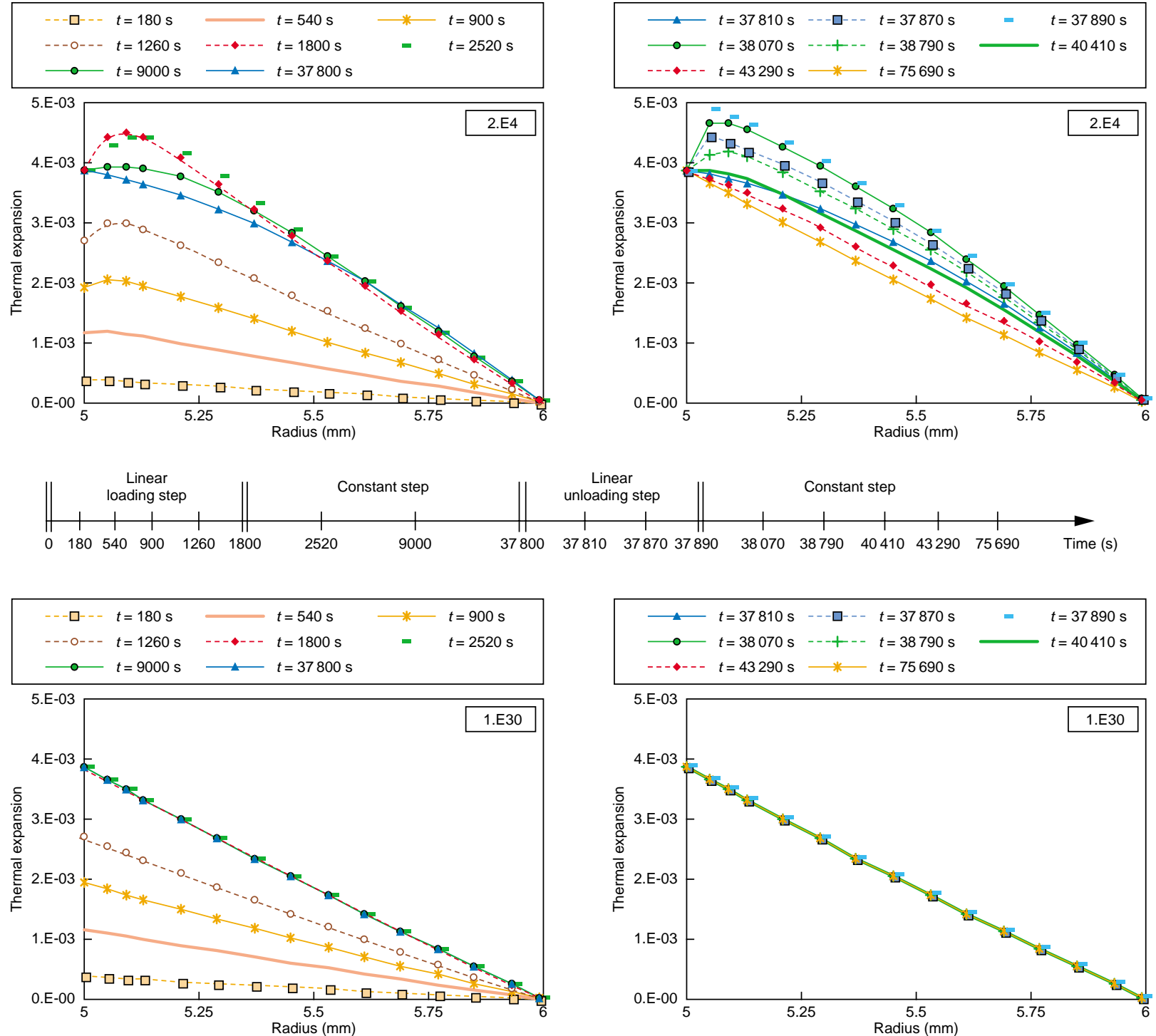

Figure 10

Profiles of thermal expansion for $k_{\mu}=2 \cdot 10^{4} \mathrm{~kg} \cdot \mathrm{s} / \mathrm{m}^{3}$ and $k_{\mu}=10^{30} \mathrm{~kg} \cdot \mathrm{s} / \mathrm{m}^{3}$. 


\section{Numerical Aspects}

Whatever the value of $k_{\mu}$, the thermal results are identical to those obtained in the previous case. A weak slowing down of the mass transport is observed. Moreover, the radial displacement, which corresponds to a decrease in volume (Fig. 12) in the uncoupled case, is reversed. A competition appears between internal pressure that compacts the sheath and diffusion as well as thermal processes that induce an expansion of the structure. The thermal (Fig. 10) and diffusion (Fig. 11) expansions have different orders of magnitude, but it is important to remind the measurement uncertainty of $\alpha_{c}$ and $\alpha_{T}$.

\subsubsection{Physical Aspects}

With these two expansion coefficients, the basic experimental results observed by IFP are recovered (contraction under the pressure effect—expansion under the absorption effect).

With the aim of differentiating the two effects, it will be necessary to know the local value of temperature. These effects are expected to be quantified during a classical permeability test. After a transient phase, the gas mass flux becomes stationary and a thermal gradient appears in the thickness owing to the " $k_{\mu}$ " coupling. Then, expansions linked to the variations of temperature and concentration induce a non-linear variation of sample thickness. A comparison between numerical predictions and local measures has to be considered.

\subsection{Introduction of $\mathrm{c}_{\mathrm{T} \mu}$}

\section{Theoretical Aspects}

Some supplementary terms appear in diffusion and thermal problems. The divergence of the thermal gradient contributes to the concentration variation. In order to conserve classical diffusion and conduction effects, $k_{\mu}$ and $c_{T \mu}$ are defined as follows:

$$
10^{4}<k_{\mu} \leq 10^{10} \text { and } 0<c_{T \mu}<10^{7}
$$

\section{Numerical Aspects}

The effects of $c_{T \mu}$ are similar whatever the value of $k_{\mu}$ but they are all the more strong as $k_{\mu}$ is small.

For small values of $c_{T \mu}\left(10^{-2} \mathrm{~kg} / \mathrm{m} \cdot \mathrm{s} \cdot \mathrm{K}\right)$, gas diffuses a little bit more slowly during the linear loading step (Fig. 13, negative absolute difference of concentration) and a little bit more quickly during the decompression step. Thus, this coefficient plays a directional role in diffusion process (absorption or desorption). It is a diminishing and/or enhancing factor. Its effect on temperature field has the same order of magnitude. During the loading step, temperature is more elevated. During the decompression step corresponding to a constant temperature on the boundary, a weak drop in temperature appears inside the material due to the acceleration of gas desorption.
For high values of $c_{T \mu}\left(10^{3} \mathrm{~kg} / \mathrm{m} \cdot \mathrm{s} \cdot \mathrm{K}\right)$, diffusion is accelerated by the $c_{T \mu} \operatorname{div}(\operatorname{grad} T)$ term which appears in the concentration evolution equation. Diffusion and conduction have the same characteristic time (Fig. 14). Concentration becomes equal to zero at the end of decompression step. Temperature increases more slowly during the two first steps. During the decompression, temperature field corresponds to a linear distribution along the radius; this is linked to the boundary conditions of temperature.

\section{Physical Aspects}

It becomes more difficult to explain the coupling effects at this stage. The new coefficient $c_{T \mu}$ appears as a multiplier in of some gradients. If within the structure considered, loading and boundary conditions generate strong and/or localised gradients, the effect of $c_{T \mu}$ will probably be more significant. This coefficient acts on the kinetics of diffusion and conduction (more or less rapid) with constant $D$ and $\Lambda$.

\subsection{Totally Coupled Case}

Now, all the coupling coefficients are considered. The study of constitutive equations leads to three threshold values of $\mathbf{d}$ :

$$
\mathbf{d}=0, \quad \mathbf{d}=\mathbf{d}_{1}=\frac{c_{T \mu}}{k_{\mu}} \text { and } \quad \mathbf{d}=\mathbf{d}_{2}=\frac{2 c_{T \mu}}{k_{\mu}}
$$

\section{Numerical Aspects}

Some problems of convergence appear often during these simulations. They could be suppressed by modifying the evolution of time step. Two results are presented in this part with the aim of underlining the effects of $\mathbf{d}$.

First, $\mathbf{d}$ is chosen equal to $\mathbf{d}_{1}$ and the results are compared to a case without $c_{T \mu}$ and $\mathbf{d}$. The thermal problem is modified and, via the thermal expansion, also the mechanical one. Some difference of concentration appears in several localized parts of the structure. The variation of temperature is all the more important as the coefficient $\left[c_{T \mu}^{2} / k_{\mu}\right]$ is close to [1/T].

The effect of $\mathbf{d}$ on each problem is then studied. For a given couple $\left(k_{\mu}, c_{T \mu}\right)$, simulations are done with different values of $\mathbf{d}$. It is difficult to associate a global effect to this coefficient because of the complicated interactions existing between mechanical, thermal and diffusion phenomena. For $\mathbf{d}$ lower to $\mathbf{d}_{1}$, an unrealistic increase in temperature appears, whereas for $\mathbf{d}$ superior to $\mathbf{d}_{2}$, temperature decreases (Fig. 15). In the same way, the effect of $d$ on concentration depends on the value of this coefficient (Fig. 16).

\section{Physical Aspects}

According to the value of $\mathbf{d}$, the effects observed are completely different. Couplings result in acceleration or slowing of mass transport and thermal transfer. This coefficient associated with $c_{T \mu}$ generates some complicated non-linear effects, which are greatly dependent on gradients. Experimental studies will make it possible to validate or not 


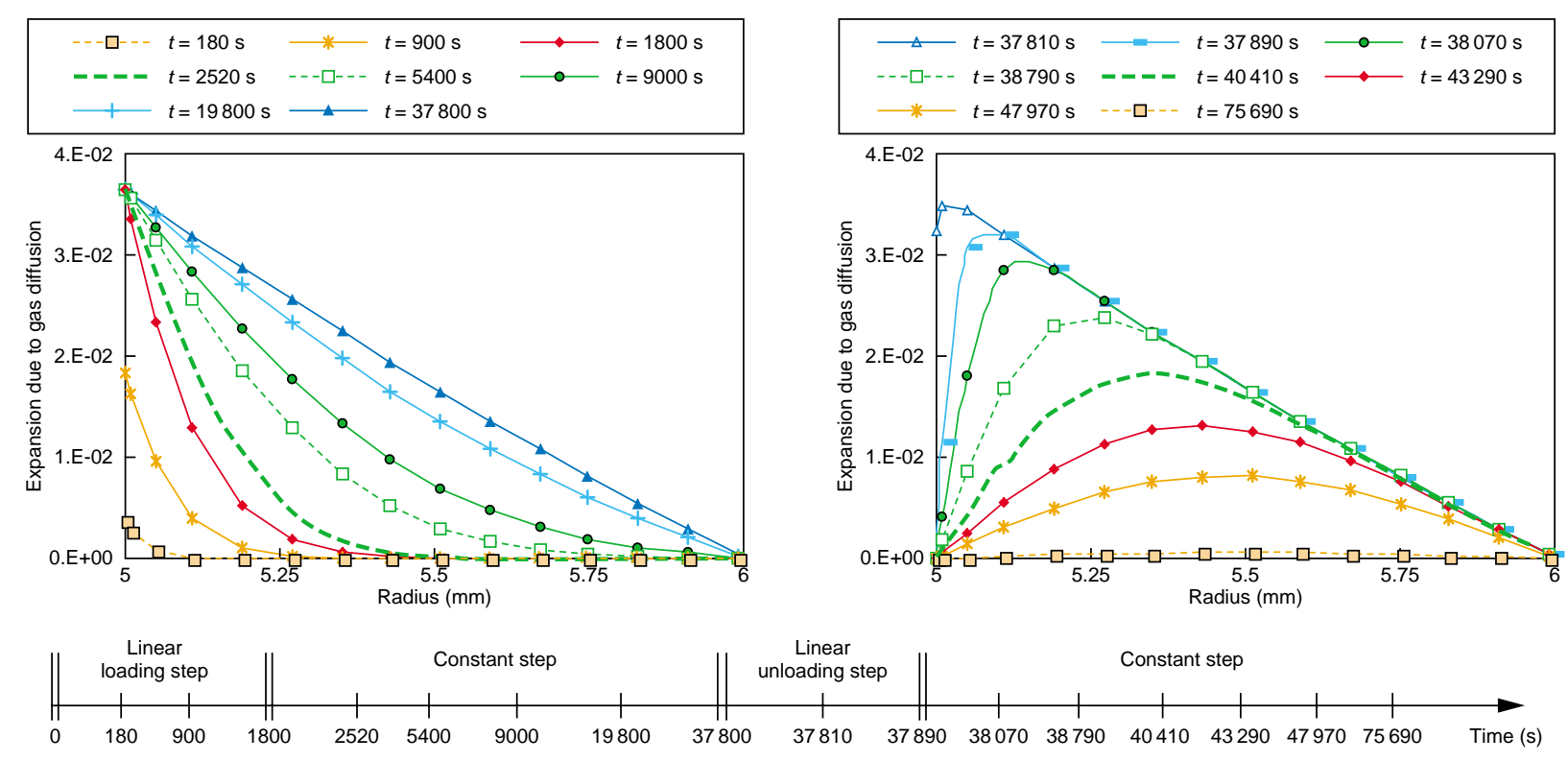

Figure 11

Profiles of expansion due to diffusion independent of $k_{\mu}$ value.

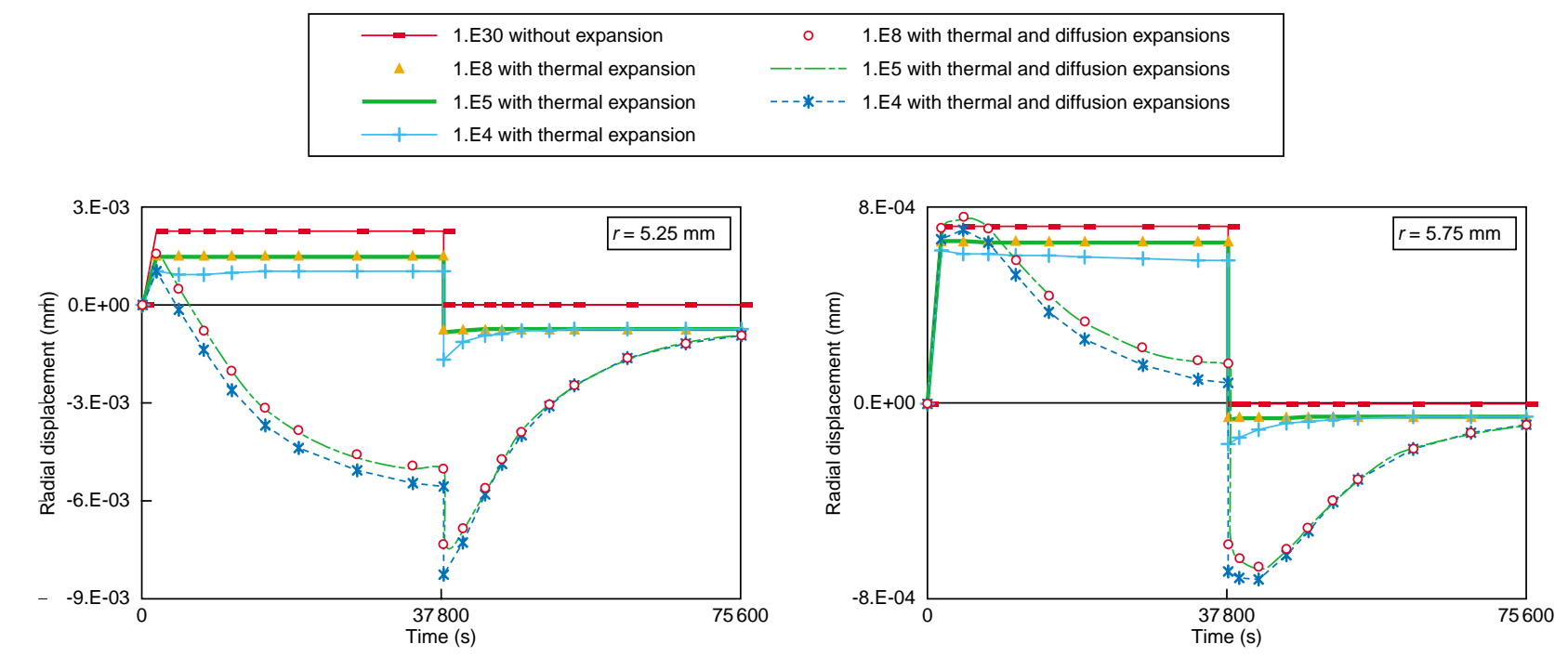

Figure 12

Effect of expansion coefficients on radial displacement. 
$\mathrm{k} \mu=10^{4} \mathrm{~kg} . \mathrm{s} / \mathrm{m}^{3}$ and $\mathrm{c}_{\mathrm{T} \mu}=10^{-2} \mathrm{~kg} /(\mathrm{m} . \mathrm{s} . \mathrm{K})$

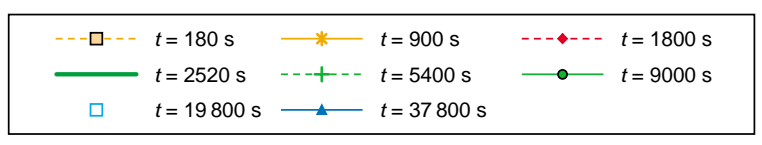
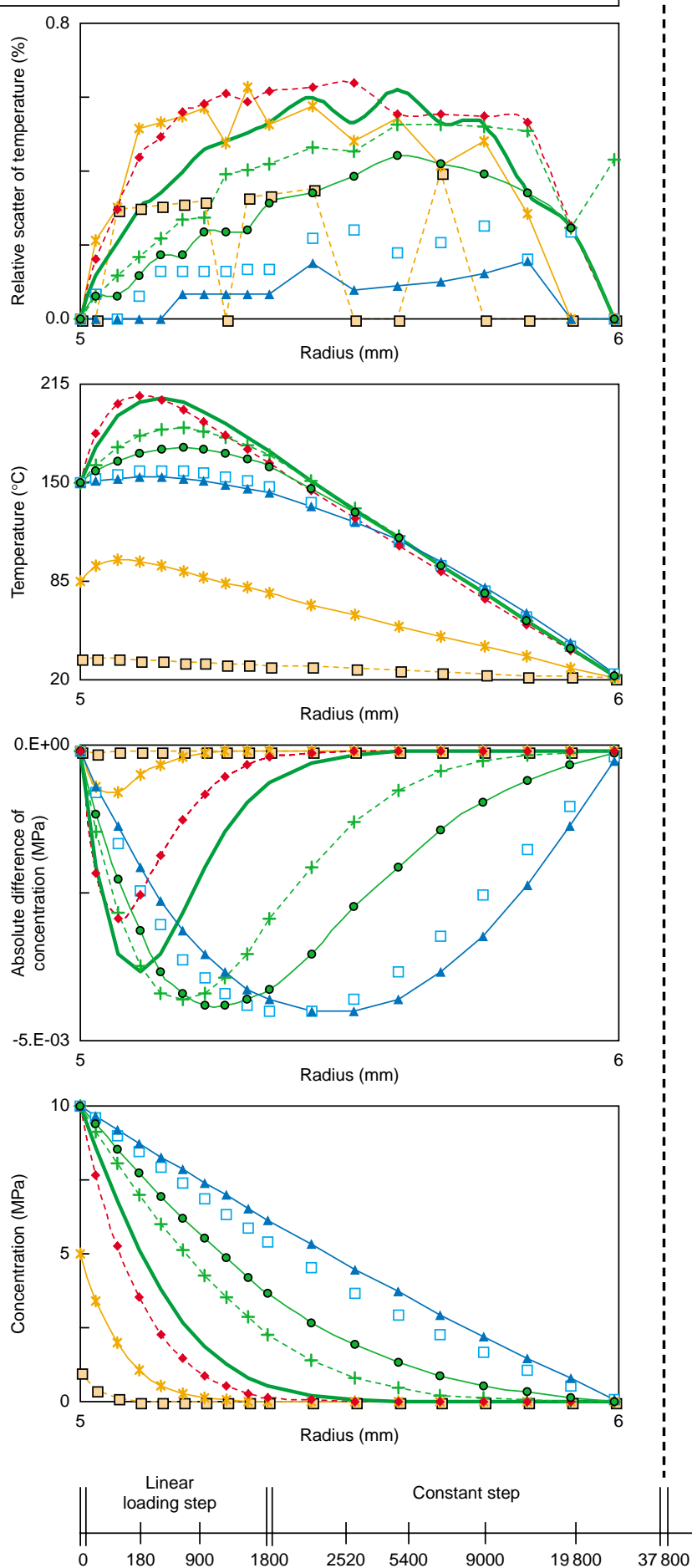
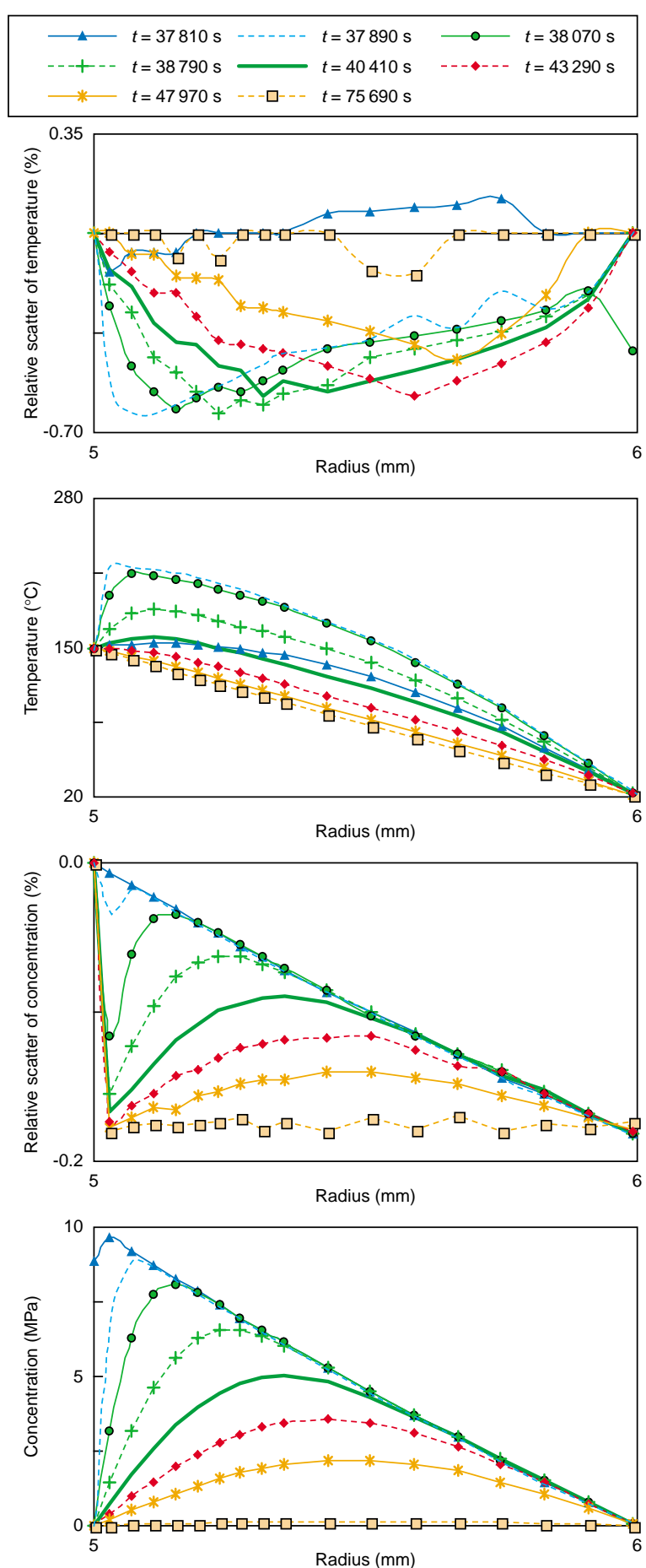

Linear $\quad$ Constant step

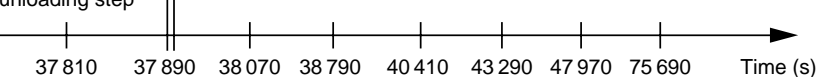

Figure 13

Effect of a small $c_{T \mu}$ value: $10^{-2} \mathrm{~kg} /(\mathrm{m} \cdot \mathrm{s} \cdot \mathrm{K})$. Comparison with a case without $c_{T \mu}$. 
$k \mu=10^{4} \mathrm{~kg} \cdot \mathrm{s} / \mathrm{m}^{3}$ and $c_{T \mu}=10^{3} \mathrm{~kg} /(\mathrm{m} \cdot \mathrm{s} \cdot \mathrm{K})$

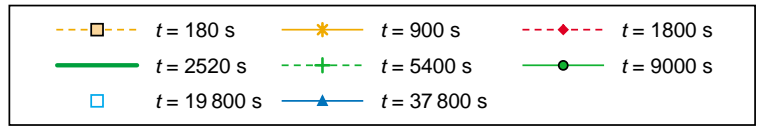
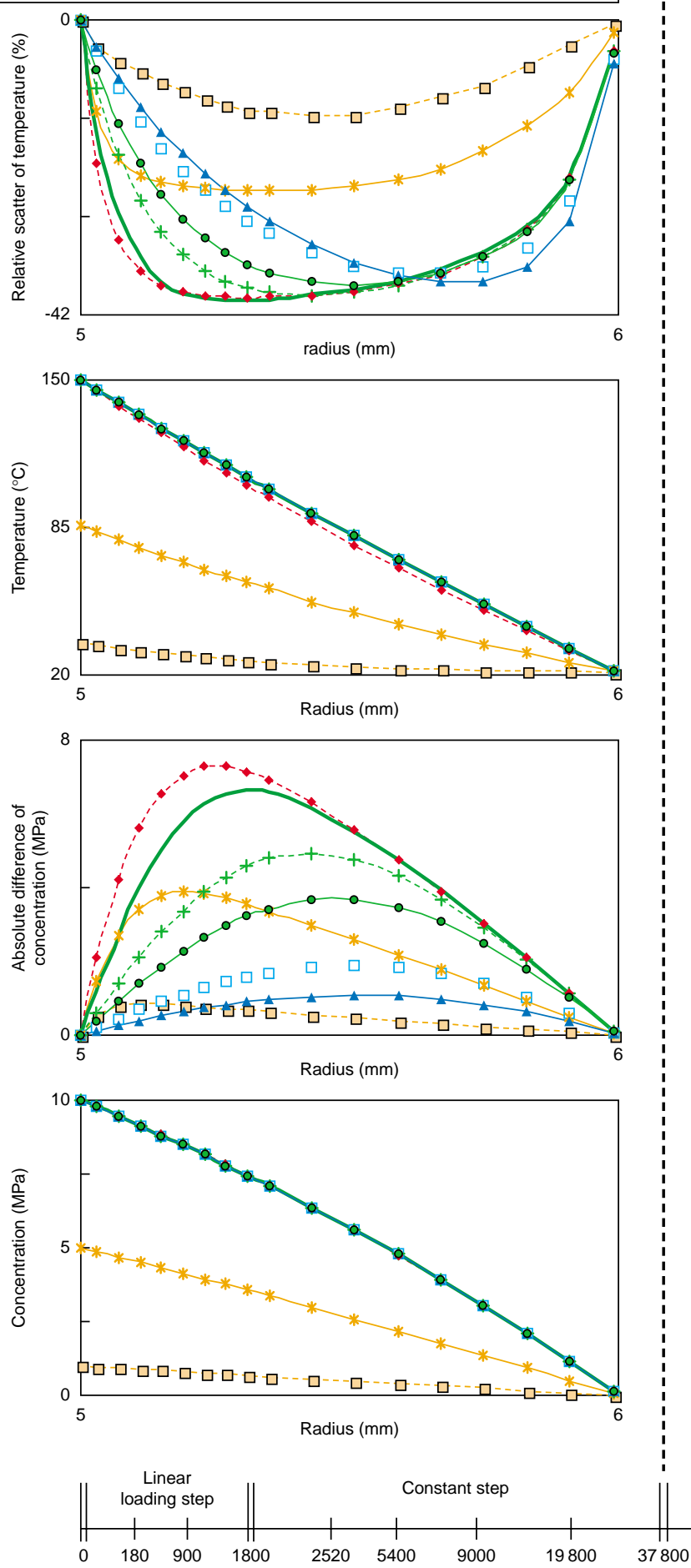
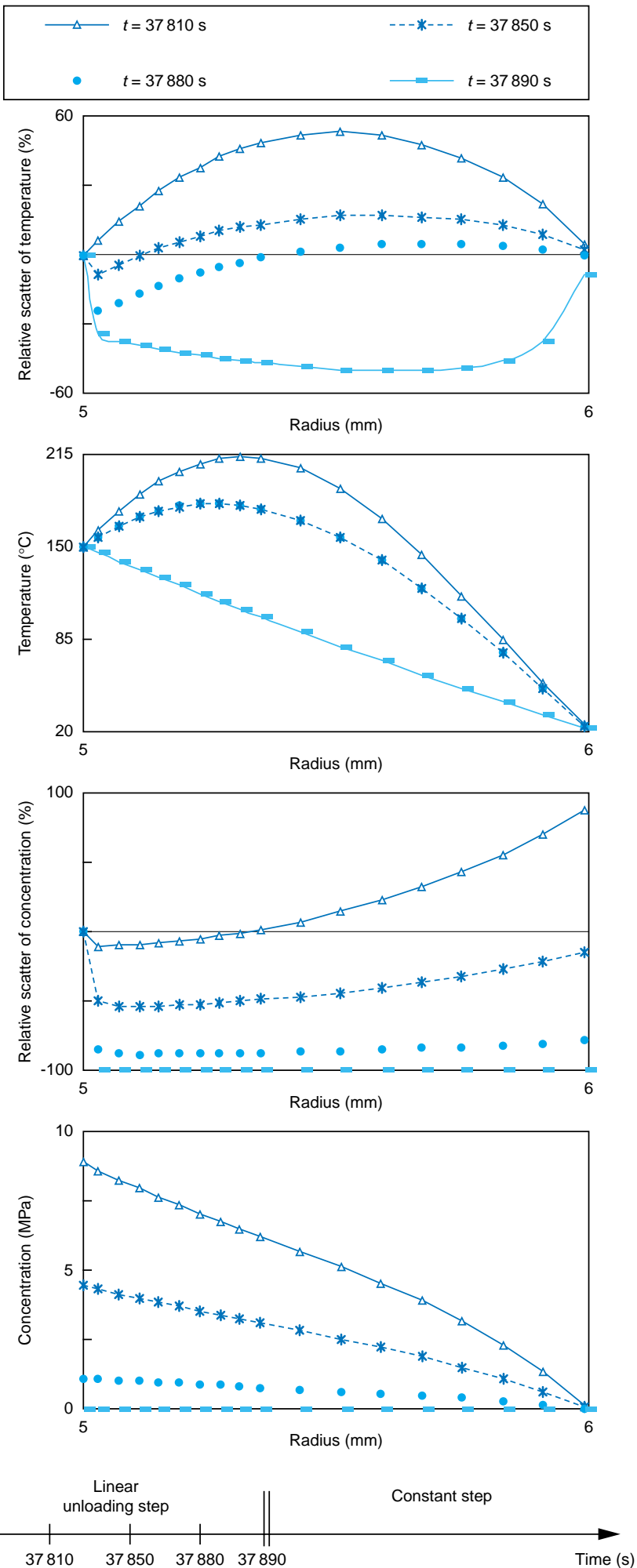

Figure 14

Effect of a high $c_{T \mu}$ value: $10^{3} \mathrm{~kg} /(\mathrm{m} \cdot \mathrm{s} \cdot \mathrm{K})$. Comparison with a case without $c_{T \mu}$. 
$k_{\mu}=10^{4} \mathrm{~kg} \cdot \mathrm{s} / \mathrm{m}^{3}, c_{T \mu}=1 \mathrm{~kg} /(\mathrm{m} \cdot \mathrm{s} \cdot \mathrm{K})$
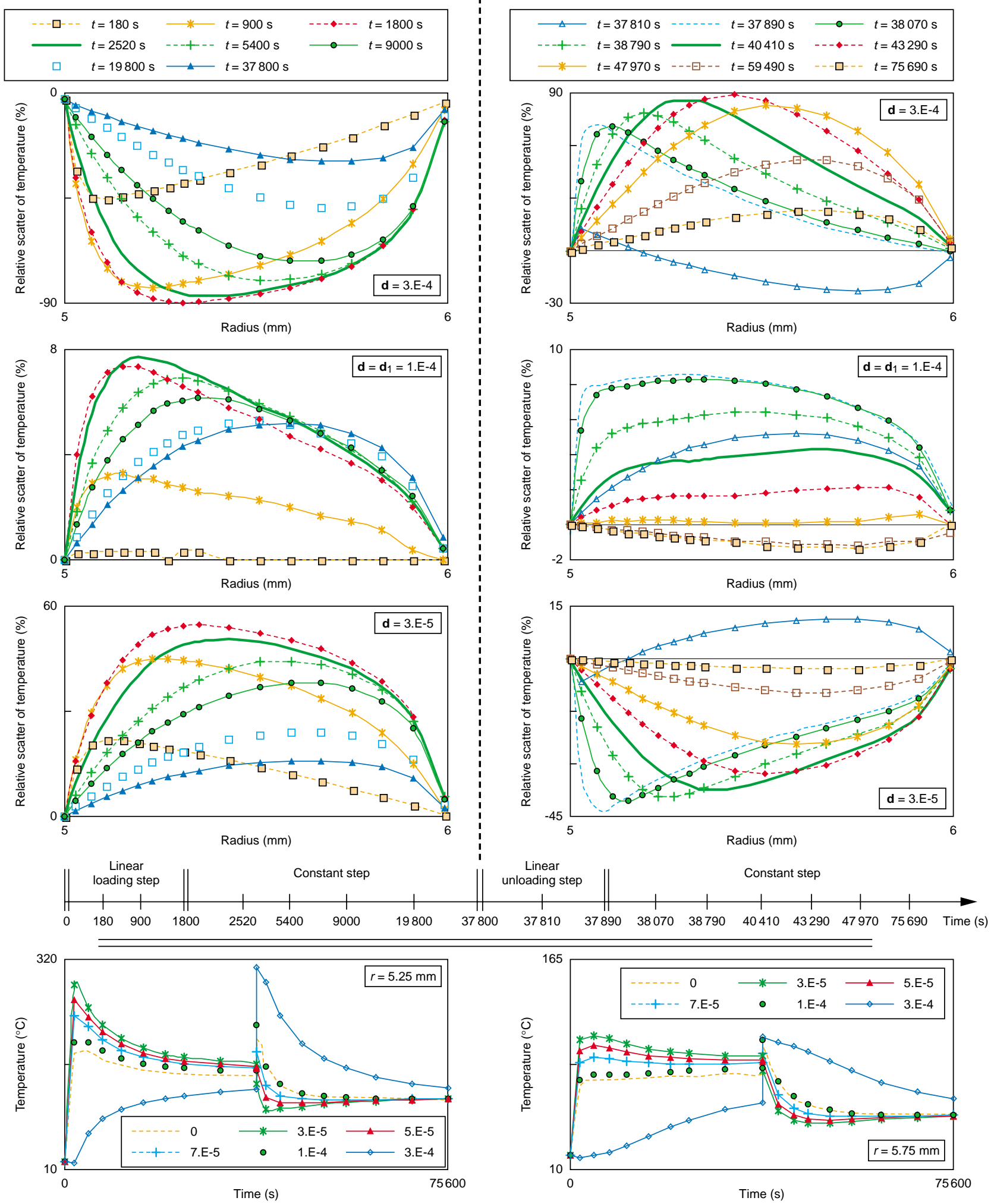

Figure 15

Effects of $c_{T \mu}$ and $\mathbf{d}$ on the thermal problem for $k_{\mu}=10^{4} \mathrm{~kg} \cdot \mathrm{s} / \mathrm{m}^{3}, c_{T \mu}=1 \mathrm{~kg} /(\mathrm{m} \cdot \mathrm{s} \cdot \mathrm{K})$ and $\mathbf{d}$ varying around $\mathbf{d}_{1}$ and $\mathbf{d}_{2}$. Comparison with the case without $c_{T \mu}$ and $\mathbf{d}$. 
$\mathrm{k} \mu=10^{4} \mathrm{~kg} \cdot \mathrm{s} / \mathrm{m}^{3}, c_{T \mu}=1 \mathrm{~kg} /(\mathrm{m} \cdot \mathrm{s} \cdot \mathrm{K})$

\begin{tabular}{|c|c|c|c|c|c|}
\hline ---口- - - & $t=180 \mathrm{~s}$ & $\longrightarrow$ & $t=900 \mathrm{~s}$ & $---\bullet--$ & $t=1800 \mathrm{~s}$ \\
\hline & $t=2520 \mathrm{~s}$ & ---+-- & $t=5400 \mathrm{~s}$ & 0 & $t=9000 \mathrm{~s}$ \\
\hline$\square$ & $t=19800 \mathrm{~s}$ & 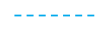 & $t=28800 \mathrm{~s}$ & 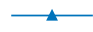 & $t=37800 \mathrm{~s}$ \\
\hline
\end{tabular}
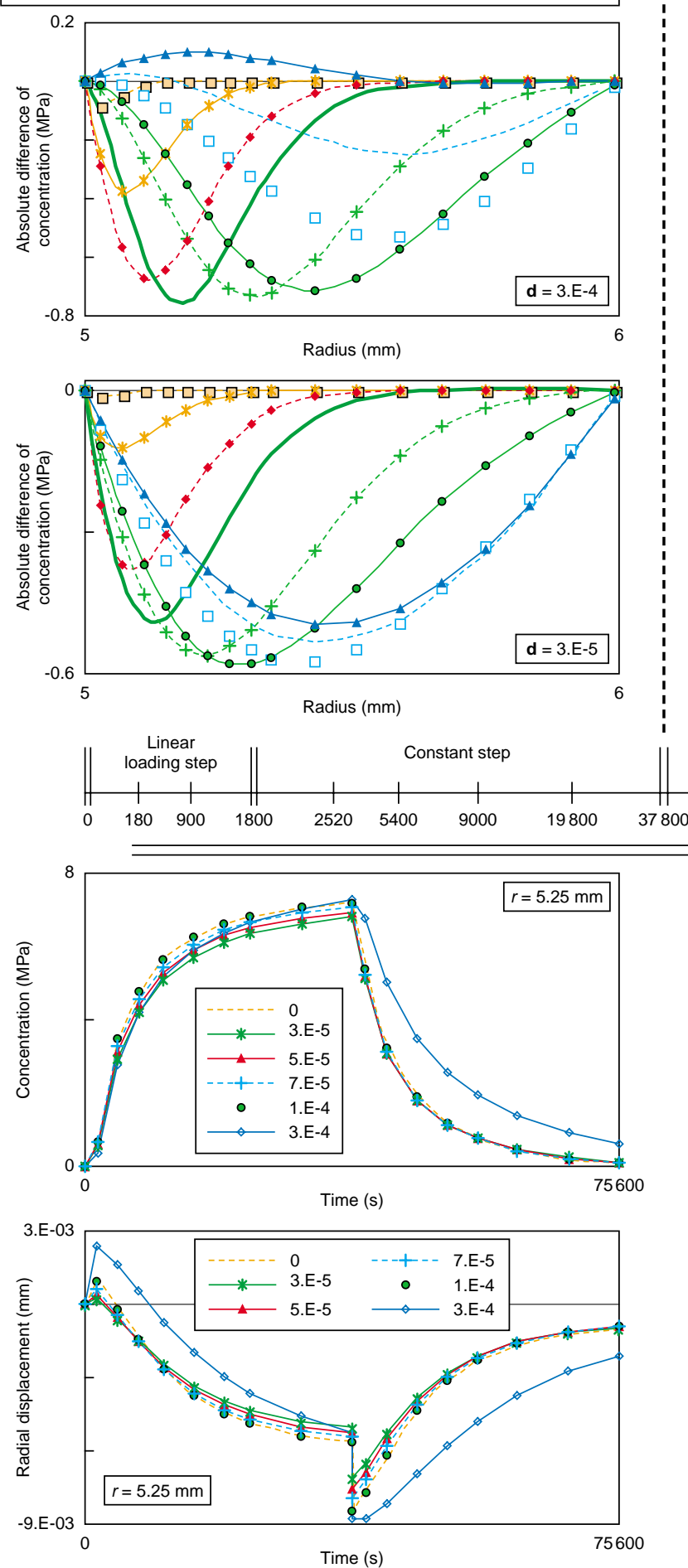
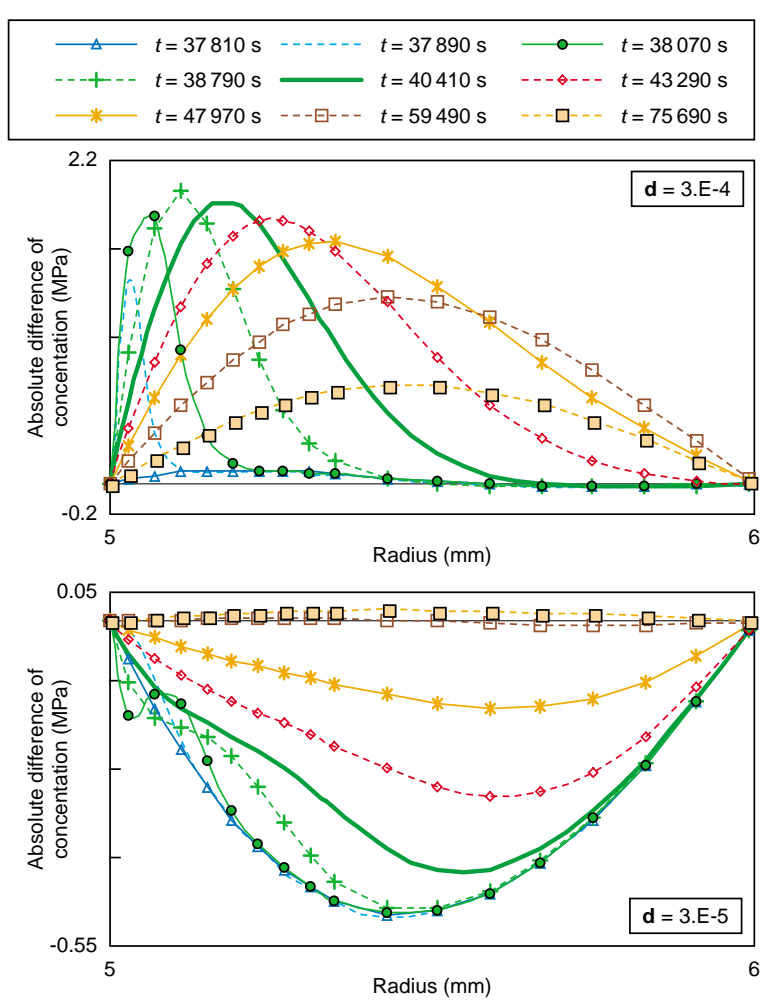

Linear

unloading step $\quad$ Constant step

$\begin{array}{lllllllll}37810 & 37890 & 38070 & 38790 & 40410 & 43290 & 47970 & 75690 & \text { Time (s) }\end{array}$
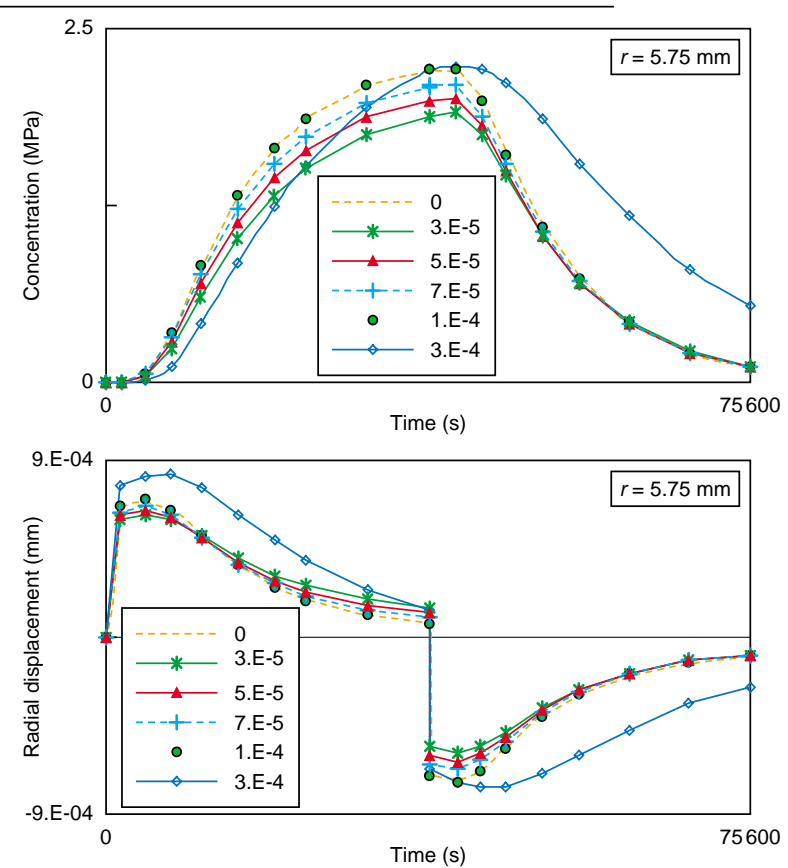

Figure 16

Effect of $c_{T \mu}$ and $\mathbf{d}$ on the mechanical and diffusion problems for $k_{\mu}=10^{4} \mathrm{~kg} \cdot \mathrm{s} / \mathrm{m}^{3}, c_{T \mu}=1 \mathrm{~kg} /(\mathrm{m} \cdot \mathrm{s} \cdot \mathrm{K})$ and $\mathbf{d}$ varying around $\mathbf{d}_{1}$ and $\mathbf{d}_{2}$. Comparison with the case without $c_{T \mu}$ and $\mathbf{d}$. 
these first results. After solving of convergence problems, a more detailed parametric study on $c_{T \mu}$ and $\mathbf{d}$ will lead to more general conclusions.

\section{CONCLUSION}

The numerical tool developed in this paper makes it possible to account for the direct couplings between mechanical, thermal and diffusion phenomena within an elastic framework. This exploratory and parametric study led to some interesting results listed below.

The coefficient $k_{\mu}$, which corresponds to the effect of diffusion on thermal evolution, is an essential parameter. Its strong impact on the field of temperatures was underlined for a large set of values of $k_{\mu}$. This parameter governs the heat generation due to the gas diffusion within the polymer. Although this view of phenomena does probably not correspond completely to the mixture theory, the effects "naturally" present in the framework of porous media are reintroduced. Indeed, the flow of a viscous fluid through the skeleton induces an increase in temperature via a mechanical effect. With the aim of improving this modelling, it will be necessary to look into the signification of such a phenomenon and to propose in the short term experimental tests to quantify the effect of $k_{\mu}$. Moreover, a simple modelling, including merely this coupling coefficient whose value is limited to a specific interval, describes de facto uncoupled behaviour.

The second interesting result appears when introducing the direct couplings with mechanics. They are represented by the more classical coefficients of expansion, $\alpha_{T}$ and $\alpha_{c}$, linked to the variations of temperature and concentration, respectively. In such a context, it becomes impossible to eliminate the effect of $k_{\mu}$; in other words the three coupling coefficients, $k_{\mu}, \alpha_{T}$ and $\alpha_{c}$, define the basic elastic modelling. Moreover, during the respective simulations of tubular structure under plane-strain conditions, strain phenomena were too slow to induce heat generation. It will be thus essential to test some different boundary-value problems in further developments.

The two further coefficients, $c_{T \mu}$ and $\mathbf{d}$, produce much more subtle effects, linked to the gradients of temperature, concentration as well as volumetric swelling strain. The effect of these parameters could probably be explained more precisely in the presence of strong gradients; depending on geometry, loading and boundary conditions inducing such configurations should be considered.

It is also important to underline that, with simple constitutive laws, i.e. developed in a linear framework (quadratic forms of state and dissipation potentials), strong nonlinearities appear due to coupling effects. Even if this work made it possible to approach some specific effects of coupling, further simulations will be necessary to capture other features of the relative phenomena.

The formulation, implemented in ABAQUS ${ }^{\mathrm{TM}}$, contains all direct couplings in the restricted mechanical framework of elasticity. As polymers exhibit a viscous behaviour for small loadings and deformations, a further extension to viscoelasticity is to be done. Within such enlarged framework, the contribution of each direct coupling parameter will be more precisely quantified and the genuine polymer characterization easier. Indeed, expressions proposed to describe the intrinsic dependencies of material characteristics (i.e. indirect couplings) are much complicated and relative experimental data not easy to exploit making necessary numerical approaches and relative parametric studies as the present one. On this basis, it is hopefully expected to propose a new protocol of characterization. A practical discernment between direct and indirect couplings effects is also to be brought forward.

\section{REFEREN CES}

Briscoe, B.J. and Liatsis, D. (1992) Internal Crack Symmetry Phenomena During Gas-Induced Rupture of Elastomers. Rubber Chemistry and Technology, 65, 350-373.

Briscoe, B.J., Kelly, C.T. and Savvas, T. (1993) Explosive Decompression Failure of Elastomers. ICHEME Research Event, 723-724.

Briscoe, B.J., Savvas, T. and Kelly, C.T. (1994) Explosive Decompression Failure of Rubbers: A Review of the Origins of Pneumatic Stress Induced Rupture in Elastomers. Rubber Chemistry and Technology, 67, 384-416.

Cailletaud, G., Aeby-Gautier, E., Berthaud, Y., Billardon, R. and Monceau, D. (2002) Problèmes multiphysiques. Couplages de la mécanique avec métallurgie/thermique/diffusion/électricité. Cours IPSI.

Campion, R.P. (1990) Explosive Decompression in Elastomers. Internal Blistering and Fracturing in Rubbers after High Pressure Exposure to Gases. Cellular Polymers, 9, 3, 206-228.

Cangémi, L. and Meimon, Y. (2001) A Two Phase Model for the Mechanical Behavior of Semicrystalline Polymers. Oil \& Gas Science and Technology - Revue de l'IFP, 56, 6, 555-580.

Coussy O. (1991) Mécanique des milieux poreux, Éditions Technip, Paris.

Cunat, C. (1991) A Thermodynamic Theory of Relaxation Based on a Distribution of Non Linear Processes. Journal of Non Crystalline Solids, 131/133, 196/812.

Dewimille, B., Martin, J. and Jarrin, J. (1993) Behaviour of Thermoplastic Polymers During Explosive Decompression in a Petroleum Environment. Journal de Physique IV, 3, 7, 15591564.

Flaconnèche, B., Martin, J. and Klopffer, M.H. (2001) Permeability, Diffusion and Solubility of Gases in Polyethylene, Polyamide 11 and Poly(vinylidene fluoride). Oil \& Gas Science and Technology - Revue de l'IFP, 56, 3, 261-278.

Gaillard-Devaux, E. (1995) Rupture du polyéthylène en température par décompression de méthane. Thèse, École nationale supérieure des mines de Paris.

Jarrin, J., Dewimille, B., Devaux, E., Martin, J. and Piques, R. (1994) Blistering of Thermoplastic Materials Used in the Petroleum Industry. SPE 28482, 203-214. 
Klopffer, M.H. and Flaconnèche, B. (2001) Transport Properties of Gases in Polymers: Bibliographic Review. Oil \& Gas Science and Technology - Revue de l'IFP, 56, 3, 223-244.

Liatsis, D. (1989) Gas Induced Rupture of Elastomers. PhD Thesis, Imperial College.

Lorge, O., Briscoe, B.J. and Dang, P. (1999) Gas Induced Damage in Poly(vinylidene fluoride) Exposed to Decompression. Polymer, 40, 2981-2991.

Onaisi, A. (1991) Linear Porothermoelastic Coupling by the Finite Element Method and Application to Petroleum Problems. Revue de l'Institut Français du Pétrole, 46, 6, 749-758.

Papon, P. and Leblond, J. (1990) Thermodynamique des états de la matière, Hermann, Paris.

Rodier-Renaud, L. (1994) Étude transitiométrique de l'influence de la pression sur les propriétés thermomécaniques des polymères. Expansibilité thermique jusqu'à $393 \mathrm{~K}$ et $300 \mathrm{MPa}$ de polyéthylènes seuls ou en interaction avec le méthane. Thèse, université Clermont-Ferrand II.

Skoczylas, F., Shahrour, I. and Henry, J.P. (1992) Étude numérique du couplage thermohydromécanique dans les roches. Influence des termes de couplage non linéaires pour un matériau isotrope linéaire. Revue de l'Institut Français du Pétrole, 47, 1, 45-55.

Tie Bi, G.R. (2000) Études expérimentales des couplages thermomécaniques par méthodes optiques et par thermographie infrarouge, applications aux polymères. Thèse, université de Poitiers.

Weiler, F.C. (1992) Fully Coupled Thermo-Poro-Elasto Governing Equations. Computational Mechanics of Porous Materials and their Thermal Decomposition, ASME, AMD, 136, $1-28$.

Final manuscript received in August 2003 